\title{
Network-Based Biomarkers for Cold Coagulation Blood Stasis Syndrome and the Therapeutic Effects of Shaofu Zhuyu Decoction in Rats
}

\author{
Shulan Su, ${ }^{1}$ Jinao Duan, ${ }^{1}$ Wenxia Cui, ${ }^{1}$ Erxing Shang, ${ }^{1}$ Pei Liu, ${ }^{1}$ Gang Bai, ${ }^{2}$ \\ Sheng Guo, ${ }^{1}$ Dawei Qian, ${ }^{1}$ and Yuping Tang ${ }^{1}$ \\ ${ }^{1}$ Jiangsu Key Laboratory for High Technology Research of TCM Formulae, Nanjing University of Chinese Medicine, \\ Nanjing 210046, China \\ ${ }^{2}$ College of Pharmacy, State Key Laboratory of Medicinal Chemical Biology, Tianjin Key Laboratory of Molecular Drug Research, \\ Nankai University, Tianjin 300071, China
}

Correspondence should be addressed to Jinao Duan; duanja@163.com

Received 25 May 2013; Accepted 1 August 2013

Academic Editor: Aiping Lu

Copyright (C) 2013 Shulan Su et al. This is an open access article distributed under the Creative Commons Attribution License, which permits unrestricted use, distribution, and reproduction in any medium, provided the original work is properly cited.

\begin{abstract}
In this study, the reverse docking methodology was applied to predict the action targets and pathways of Shaofu Zhuyu decoction (SFZYD) bioactive ingredients. Furthermore, Traditional Chinese Medicine (TCM) cold coagulation blood stasis (CCBS) syndrome was induced in female Sprague-Dawley rats with an ice-water bath and epinephrine, and SFZYD was used to treat CCBS syndrome. A metabolomic approach was used to evaluate changes in the metabolic profiles and to analyze the pharmacological mechanism of SFZYD actions. Twenty-three potential protein targets and 15 pathways were discovered, respectively; among these, pathways are associated with inflammation and immunological stress, hormone metabolism, coagulation function, and glycometabolism. There were also changes in the levels of endogenous metabolites of LysoPCs and glucuronides. Twenty endogenous metabolites were identified. Furthermore, the relative quantities of 6 endogenous metabolites in the plasma and 5 in the urine were significantly affected by SFZYD $(P<0.05)$. The pharmacological mechanism of SFZYD was partially associated with glycerophospholipid metabolism and pentose and glucuronate interconversions. In conclusion, our findings demonstrated that TCM CCBS pattern induced by ice water and epinephrine was complex and related to multiple metabolic pathways. SFZYD did regulate the TCM CCBS by multitargets, and biomarkers and SFZYD should be used for the clinical treatment of CCBS syndrome.
\end{abstract}

\section{Introduction}

Traditional Chinese medicine (TCM) is guided by the theory of traditional Chinese medical science for clinical application. TCM can be characterized as being holistic, with an emphasis on regulation of the integrity of the human body and the interaction between individuals and their environment. Multiple natural therapeutic methods for patient's management were applied in TCM; among these, the herbal formula was the most typical treatment. Most herbal medicines are prescribed in combination to obtain synergistic effects or diminish possible adverse reactions [1]. This medical approach has played on increasingly important role in evidence-based personalized medicine, which is a new trend and a hot research topic of medical development.

Based on the theory of TCM, which is rooted in the philosophy of treating the entire body as a whole, multipathogenic factors, such as cold coagulation, Qi stagnation, and blood insufficiency, are considered to be the main causes of many diseases or syndromes. In TCM, there are several different disharmony patterns (named ZHENG) such as the syndrome of blood stasis, syndrome of Qi stagnation, and Yin/Yang deficiency syndrome [2]. The TCM ZHENG as a diagnostic approach may provide an invaluable guidance for therapeutic choices and personalized disease management, not only in traditional medical practices but also in modern healthcare systems [3]. 
Cold coagulation blood stasis (CCBS) is primarily induced by cold-evil, and as a subtype of blood stasis syndrome, it is a critical pattern for many diseases, especially in women. The pathologic mechanisms of CCBS syndrome were recently found to be related to the changes in hemorheological properties including high-blood viscosity, increased erythrocyte aggregation, increased blood sedimentation, decreased erythrocyte deformability, decreased hematocrit, microcirculation disturbance, and coagulant function abnormality [4]. Further research revealed that blood stasis syndrome is closely related to inflammatory factors and the immune response. Inflammatory- and immune-related genes are remarkably dominant in the gene expression profiles of blood stasis syndrome, which may explain the functions of inflammation and the immune response in the occurrence and development of this syndrome [5]. Moreover, it was reported that the occurrence of vasomotor dysfunction in the ovaries and the levels of reproductive hormone decrease in patients with blood stasis syndrome [6], but the complex pathological mechanisms and metabolite profiling changes in CCBS syndrome remain incompletely elucidated.

The famous Chinese herbal formula, Shaofu Zhuyu decoction (SFZYD), which was originally identified by the "Correction of Errors in Medical Classics" compiled by Qingren Wang in the Qing dynasty (A.D. 1830), was utilized in the clinic for approximately 200 years to treat blood stasis syndrome in gynecological diseases such as dysmenorrhea, amenorrhea, or menoxenia. SFZYD is considered an effective prescription for the treatment of CCBS syndrome, and it is composed of ten crude drugs: Angelicae sinensis Radix, Chuanxiong Rhizoma, Cinnamomi Cortex, Foeniculi Fructus, Zingiberis Rhizoma, Myrrha, Trogopterpri Faeces, Typhae Pollen, Paeoniae Radix Rubra, and Corydalis Rhizoma [7]. In the clinic, SFZYD was reported to have over $90 \%$ efficacy for the treatment of primary dysmenorrhea with CCBS syndrome $[8,9]$. However, the therapeutic mechanisms of SFZYD are still unknown and require a comprehensive investigation.

Metabolomics, which is based on the dynamic changes of low molecular weight metabolites in organisms, indicates the overall physiological status in response to pathophysiological stimuli or genetic, environmental, or lifestyle factors [10]. Metabolites often mirror the end result of genomic and protein perturbations in a disease, and they are most closely associated with phenotypic changes. Furthermore, the pathogenesis of diseases and the mechanisms of action for therapies can be elucidated by identifying biomarkers, analyzing the metabolic pathway, discovering drug-target interactions, and so on. Recently, metabolic profiling has attracted great interest for biomarker discovery and for assessing the holistic effects of many therapeutics used in TCM [11-16].

Network pharmacology, a system biology-based methodology, is a new approach to highlight a holistic thinking and systematical theory of interactions among drugs, targets, and diseases, which is also shared with TCM theory. The remarkable feature of network pharmacology is the "multicomponent therapeutics, network target" [17-19]. In the network pharmacology-based drug research, a biological network of a disease and a pharmacological network of the candidates are established. So, the network pharmacology applied to TCM research would provide a novel methodology and opportunity for screening bioactive ingredients, synergistic drug combinations [20], and biomarkers, revealing mechanisms of action and exploring scientific evidence of herbs [21] or herbal formulae on the basis of complex biological systems [22].

Here, for the first time, we studied the network targets and pathway of SFZYD bioactive ingredients. Furthermore, the plasma and urine metabolomics of cold coagulation blood stasis syndrome, which was induced by epinephrine and cold evil, and the therapeutic effects of SFZYD in rats were investigated. We used the ultra-high-performance liquid chromatography in tandem with a time-of-flight mass spectrometry (UHPLC-TOF/MS) based metabolomic approach to elucidate the metabolic profiles and phenotype changes between normal rats and model rats and to identify potential biomarkers. A multivariate statistical analysis was used to investigate the pathological variations of cold coagulation blood stasis syndrome and to explore the therapeutic effects of SFZYD.

\section{Materials and Methods}

2.1. Materials. Ten crude herbs, Angelicae sinensis Radix, Chuanxiong Rhizoma, Paeoniae Radix Rubra, Cinnamomi Cortex, Foeniculi Fructus, Zingiberis Rhizoma, Myrrha, Trogopterpri Faeces, Typhae Pollen, and Corydalis Rhizoma, were purchased from Minxian (Gansu), Pengzhou (Sichuan), Chifeng (Neimeng), Yulin (Guangxi), Wuwei (Gansu), Yulin (Guangxi), Guangdong, Changzhi (Shanxi), Jiangsu (Yixing), and Songyang (Zhejiang), respectively. The corresponding author authenticated all of the raw materials, and the herbal drugs were verified according to the Chinese pharmacopoeia (Chinese pharmacopoeia, 2010). The voucher specimens (no. NJUTCM-20110818-20110827) were deposited in the Jiangsu Key Laboratory for TCM Formulae Research of Nanjing University of Chinese Medicine.

HPLC grade acetonitrile was purchased from Tedia (Fairfield, $\mathrm{OH}, \mathrm{USA}$ ), and AR grade formic acid was purchased from the Shanghai Reagent Company (Shanghai, China). Ultrapure water for UHPLC analysis was prepared using a Millipore water purification system (Millipore, Milford, MA, USA) and filtered with $0.22 \mu \mathrm{m}$ membranes prior to use. Sodium citrate (no. 20071107) and epinephrine hydrochloride (no. 0808231) were obtained from Tianjin Biochem Pharmaceutical CO., LTD., and Tianjin King York Amino Acid CO., LTD., respectively.

\subsection{Preparation of SFZYD Extract. Angelicae sinensis Radix,} Chuanxiong Rhizoma, Paeoniae Radix Rubra, Cinnamomi Cortex, Foeniculi Fructus, Zingiberis Rhizoma, Myrrha, Trogopterpri Faeces, Typhae Pollen, and Corydalis Rhizoma were mixed at a weight ratio of $3: 1: 2: 1: 0.5: 2: 1: 3: 1: 1$, respectively. The mixture $(1.5 \mathrm{~kg})$ was decocted with $15 \mathrm{~L}$ of water for $2 \mathrm{~h}$. The filtrates were collected, and the residues 
were decocted in $12 \mathrm{~L}$ of water for $1.5 \mathrm{~h}$. The filtrates from each decoction were combined and concentrated to $1.5 \mathrm{~L}$ at $70^{\circ} \mathrm{C}$. The concentrated solution was dried with a vacuum, and $28.9 \mathrm{~g}$ extract of Shaofu Zhuyu decoction (SFZYD) was produced.

\subsection{Network Targets and Pathway Prediction of SFZYD Bioac-} tive Ingredients. Based on our previous studies [23, 24], the ten compounds absorbed into blood were selected to predict the biological targets. The mol2 files of ten compounds are imported into PharmMapper database (http://59.78.96 $.61 /$ pharmmapper/) to predict the targets, then the target numbers were entered into the database (http://www.uniprot .org/, http://bioinfo.capitalbio.com/mas3/), and the Kyoto Encyclopedia of Genes and Genomes (KEGG) database (http://www.genome.jp/kegg/) was used to annotate and analyze the pathway. The chemical structures of ten compounds from SFZYD were shown in Figure 1.

2.4. Animal Handling Procedure and Drug Treatment. Female Sprague-Dawley (SD) rats $(180 \pm 10 \mathrm{~g})$ were purchased from Nanjing University of Chinese Medicine (rodent license no. SCXK 20080033). The rats were housed under standard laboratory conditions, and food and tap water were provided ad libitum. Experimental procedures were carried out in accordance with the Guide for the Care and Use of Laboratory Animals, and before the animal experiments were carried out, the procedures were approved by the Laboratory Animal Center of Nanjing University of Chinese Medicine.

The experimental groups $(n=6)$ were as follows: (1) normal control (NC), (2) cold- and epinephrine-induced CCBS syndrome (CCBS), and (3) CCBS model rats with SFZYD treatment. The model rats were put into ice water $\left(0^{\circ} \mathrm{C} \sim 1^{\circ} \mathrm{C}\right)$ for $5 \mathrm{~min}$ daily for 7 days. On the 8 th day, they received two subcutaneous injections of hypodermic epinephrine $\left(1 \mathrm{mg} \cdot \mathrm{kg}^{-1}\right)$ at 4 -hour intervals. Simultaneously, model rats were administered SFZYD $\left(10.08 \mathrm{~g} \mathrm{~kg}^{-1} ; 10\right.$ times the clinical dose for humans) via gastric irrigation once daily for 7 days. The dose of SFZYD was chosen based on the clinical application dosage of $45 \mathrm{~g} /$ day $/ 60 \mathrm{~kg}$ body weight. The rats in the normal control group were treated with an equal volume of distilled water as a vehicle control.

2.5. Samples Collection and Preparation. Blood samples were collected in heparinized tubes on the 8th day after the injection of epinephrine. They were then anticoagulated in natrium citricum, centrifuged at $15000 \times \mathrm{g}$ for $10 \mathrm{~min}$, and stored at $-20^{\circ} \mathrm{C}$ until analysis. Urine samples were collected in 12-hour intervals every day for 10 days, then centrifuged at $15000 \times \mathrm{g}$ for $10 \mathrm{~min}$ and stored at $-20^{\circ} \mathrm{C}$ until analysis.

Two hundred microliters of plasma was added to $600 \mu \mathrm{L}$ of acetonitrile, and this mixture was vortexed for $30 \mathrm{~s}$ and centrifuged at $15,000 \times \mathrm{g}$ for $10 \mathrm{~min}$ to obtain the supernatant. Prior to analysis, the urine samples were thawed at room temperature and centrifuged at $15000 \times \mathrm{g}$ for $10 \mathrm{~min}$. The supernatant liquid $(1 \mathrm{~mL})$ was added to $3 \mathrm{~mL}$ of acetonitrile, vortex mixed for $30 \mathrm{~s}$, and centrifuged at $15000 \times \mathrm{g}$ for $10 \mathrm{~min}$ to obtain the supernatant. The samples were processed and analyzed in a random order.

The plasma and urine supernatants were removed and evaporated to dryness in a $40^{\circ} \mathrm{C}$ water bath under a gentle stream of nitrogen. The residues were reconstituted in $200 \mu \mathrm{L}$ mobile phase of $70 \%$ acetonitrile-water solution, centrifuged at $15000 \times \mathrm{g}$ for $5 \mathrm{~min}$, and filtered through a $0.22 \mu \mathrm{m}$ membrane filter. The filtrates were transferred to an autosampler vial and stored at $4^{\circ} \mathrm{C}$. A $5 \mu \mathrm{L}$ aliquot of each plasma or urine sample was injected for LC/MS analysis. The samples were analyzed in a random order.

2.6. Model Assessment. The hemorheology indexes, coagulation function, and metabolic profiling changes were calculated to evaluate the success of the CCBS syndrome model in rats. The hemorheology indexes of whole blood viscosity and plasma viscosity were measured according to a previously described method [25]. The coagulation function index, including thrombin time (TT), prothrombin time (PT), activated partial thromboplastin time (APTT), and fibrinogen (FIB), was determined to assess the CCBS syndrome model. Thrombin time (TT) was determined using a coagulometer (Model LG-PABER-I, Steellex Co., China). Shortly after adding the thrombin solution, the coagulometer was started and TT was recorded. To establish the standard curve of TT and thrombin concentration, TT was determined by incubating $40 \mu \mathrm{L}$ of plasma for $3 \mathrm{~min}$ at $37^{\circ} \mathrm{C}$, and this was followed by the addition of $40 \mu \mathrm{L}$ of a thrombin solution (different concentrations in Tris- $\mathrm{HCl}$ butter, $\mathrm{pH}$ 7.4) and $20 \mu \mathrm{L}$ of solvent for $3 \mathrm{~min}$ at $37^{\circ} \mathrm{C}$. TT was examined using the previously described method and converted into thrombin concentration using the indicated regression equation. Prothrombin time (PT), activated partial thromboplastin time (APTT), and fibrinogen content (FIB) were examined with commercial kits, following the manufacturer's instructions with slight modification. PT was determined by incubating $40 \mu \mathrm{L}$ of plasma solution for $3 \mathrm{~min}$ at $37^{\circ} \mathrm{C}$, followed by the addition of $40 \mu \mathrm{L}$ of thromboplastin agent and $20 \mu \mathrm{L}$ of sample. APTT was determined by incubating $10 \mu \mathrm{L}$ of the sample solution and $50 \mu \mathrm{L}$ of plasma with $50 \mu \mathrm{L}$ of APTTactivating agents for $3 \mathrm{~min}$ at $37^{\circ} \mathrm{C}$, followed by the addition of $50 \mu \mathrm{L}$ of $\mathrm{CaCl}_{2}$. FIB was determined by incubating $10 \mu \mathrm{L}$ of plasma with $90 \mu \mathrm{L}$ of imidazole buffer for $3 \mathrm{~min}$ at $37^{\circ} \mathrm{C}$, followed by the addition of $50 \mu \mathrm{L}$ of FIB agent and $10 \mu \mathrm{L}$ of the sample solution. 5 -HT, NA, and $\beta$-EP were determined according to the methods of ELISA kits described.

2.7. UPLC-QTOF/MS and UPLC-QqQ/MS Analysis. Chromatography was performed on an AcQuity UHPLC system (Waters Corp., Milford, MA, USA) with a conditioned autosampler at $4^{\circ} \mathrm{C}$. The separation was carried out on an AcQuity UHPLC BEH $\mathrm{C}_{18}$ column $(50 \mathrm{~mm} \times 2.1 \mathrm{~mm}$ i.d., $1.7 \mu \mathrm{m}$; Waters Corp., Milford, MA, USA), which was maintained at $35^{\circ} \mathrm{C}$. The mobile phase consisted of $0.1 \%$ formic acid ( $\mathrm{HOOCH})$ in water as solvent $\mathrm{A}$ and acetonitrile $(\mathrm{ACN})$ as solvent $\mathrm{B}$. The gradient conditions of the mobile phase were as follows: 0 4 $\mathrm{min}, \mathrm{A}: 98 \% \sim 85 \%$; $49 \mathrm{~min}, \mathrm{~A}$ : 85\% 70\%; 9 12 $\mathrm{min}, \mathrm{A}: 70 \% \sim 65 \%$; 12 15 $\mathrm{min}, \mathrm{A}: 65 \%$; 15 
<smiles>COc1cc(/C=C/C(=O)O)ccc1O</smiles><smiles>O=C(O)/C=C/c1ccc(O)c(O)c1</smiles><smiles>O=C(O)c1ccc(O)c(O)c1</smiles>

3<smiles>COc1cc(C(=O)O)ccc1O</smiles><smiles>O=c1c(O)c(-c2ccc(O)c(O)c2)oc2cc(O)cc(O)c12</smiles><smiles>COc1ccc(-c2oc3cc(O)cc(O)c3c(=O)c2O)cc1O</smiles>

5

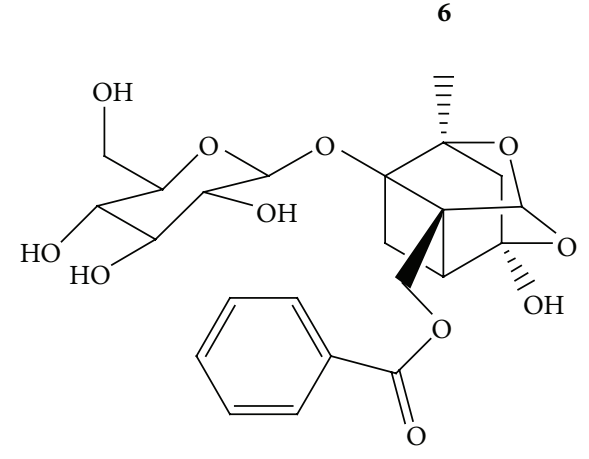

8<smiles>CCC/C=C1\CC(=O)C2=C1CC[C@H](O)[C@H]2O</smiles>

10

FIGURE 1: The chemical structures of the ten target compounds ((1) ferulic acid; (2) caffeic acid; (3) protocatechuic acid; (4) vanillic acid; (5) quercetin; (6) isorhamnetin; (7) typhaneoside; (8) paeoniflorin; (9) tetrahydropalmatine; (10) senkyunolide I).

$18 \mathrm{~min}, \mathrm{~A}: 65 \% \sim 50 \% ; 18 \sim 21 \mathrm{~min}, 50 \% \sim 25 \% ; 21 \sim 22 \mathrm{~min}, 25 \% \sim$ 20\%; 22 26 $\mathrm{min}, 20 \% \sim 5 \%$; 26 28 $\mathrm{min}, 5 \%$; and 29 31 $\mathrm{min}$, $98 \%$. The flow rate was $0.40 \mathrm{~mL} \mathrm{~min}^{-1}$, and the sample injection volume was $2 \mu \mathrm{L}$.

Mass spectrometric detection was carried out on an AcQuity Synapt mass spectrometer equipped with an electrospray ionization (ESI) interface (Waters, Milford, MA, USA). The ESI source was operated in negative and positive ionization modes. The ionization source conditions were as follows: capillary voltage of $3.0 \mathrm{kV}$, source temperature of $120^{\circ} \mathrm{C}$, and desolvation temperature of $350^{\circ} \mathrm{C}$. The sampling cone voltage was set to $30 \mathrm{~V}$, the extraction cone voltage was
$2.0 \mathrm{~V}$ (for plasma sample) or $0.7 \mathrm{~V}$ (for urine sample), the trap collision energy was $6.0 \mathrm{~V}$, the transfer collision energy was $4.0 \mathrm{~V}$, the trap gas flow was $1.50 \mathrm{~mL} \mathrm{~min}^{-1}$, and the ion energy was $1.0 \mathrm{~V}$. Nitrogen and argon were used as cone and collision gases, respectively. The cone and desolvation gas flow were 50 and $600 \mathrm{~L} \mathrm{~h}^{-1}$, respectively. The scan time was $0.5 \mathrm{~s}$ (for plasma sample) or $0.2 \mathrm{~s}$ (for urine sample), and an interval scan time of $0.02 \mathrm{~s}$ was used throughout, with a collision energy of $6 \mathrm{eV}$. The mass spectrometric data were collected from $\mathrm{m} / z 100$ to 1000 in centroid mode. Leucineenkephalin was used as the lock mass, generating an $[\mathrm{M}+\mathrm{H}]^{+}$ ion $(m / z 556.2771)$ and $[\mathrm{M}-\mathrm{H}]^{-}$ion $(m / z 554.2615)$ at a 
concentration of $200 \mathrm{pg} \mathrm{mL}^{-1}$ and a flow rate of $100 \mu \mathrm{L} \mathrm{min}^{-1}$. Dynamic range enhancement was applied throughout the MS experiment to ensure that accurate mass measurements were obtained over a wider dynamic range.

2.8. Metabolomic Data Processing and Multivariate Analysis. UPLC/MS data were detected and noise-reduced in both the UPLC and MS domains such that only true analytical peaks were selected for further processing by the software. A list of the peak intensities detected was then generated for the first chromatogram using the Rt- $m / z$ data pairs as identifiers. The resulting normalized peak intensities form a single matrix, with Rt- $m / z$ pairs for each file in the dataset. All processed data from each chromatogram were normalized and Paretoscaled prior to the multivariate statistical analysis.

All data from the plasma and urine samples were processed using the MarkerLynx application manager for MassLynx 4.1 and MarkerLynx software (Waters Corp., Milford, USA). The intensity of each ion was normalized with respect to the total ion count to generate a data matrix consisting of the retention time, $m / z$ value, and normalized peak area. The multivariate data matrix was analyzed using $E Z$ info software (Waters Corp., Milford, USA). Unsupervised segregation was examined with a principal component analysis (PCA) using Pareto-scaled data. A partial least squared discriminant analysis (PLS-DA) and an orthogonal partial least-squared discriminant analysis (OPLS-DA) were used to identify the various metabolites responsible for the separation between the model and normal groups. Potential biomarkers of interest were extracted from the S-plots that were constructed following the OPLS-DA analysis, and the biomarkers were chosen based on their contribution to the variation and correlation within the dataset.

An internal 5-fold cross-validation was carried out to estimate the performance of the PLS-DA models. The calculated $R^{2} Y_{\text {(cum) }}$ estimates how well the model represents the fraction of explained Y-variation, and $Q_{(\text {cum })}^{2}$ estimates the predictive ability of the model. Models are considered excellent when the cumulative values of $R^{2} Y$ and $Q^{2}$ are greater than 0.8 . In addition to cross-validation, 200 permutation tests were also performed to validate the model. The variable importance in the projection (VIP) value is a weighted sum of squares of the PLS weights that reflects the relative contribution of each $X$ variable to the model. The variables with VIP > 1 were considered to be influential for sample separation in the score plots generated from PLS-DA analysis [26]. Ultimately, different metabolic features associated with the model group and the SFZYD treatment group were obtained based on cutoff points of both VIP values from a 5 -fold cross-validated PLS-DA model and critical $P$ values from a univariate analysis. In addition, the corresponding fold change was calculated to show the degree of variation in metabolite levels between groups.

2.9. Biomarker Identification and Construction of the Metabolic Pathway. The identities of the potential biomarkers were confirmed by comparing their mass spectra and chromatographic retention times with the available reference standards and a full spectral library containing MS/MS data obtained in the positive and/or negative ion modes. The Mass Fragment application manager (Waters MassLynx v4.1, Waters Corp., Milford, USA) was used to facilitate the MS/ MS fragment ion analysis through the use of chemically intelligent peak-matching algorithms. This information was then used to search multiple databases, either inhouse or using online data sources, including ChemSpider database (http://www.chemspider.com), Mass Bank (http:// www.massbank.jp/), PubChem (http://ncbi.nlm.nih.gov/), and MetFrag (http://msbi.ipb-halle.de/MetFrag/).

To identify the affected metabolic pathways, a construction, interaction, and pathway analysis of potential biomarkers was performed using MetPA (http://metpa.metabolomics .ca./MetPA/faces/Home.jsp) based on several database sources, including the KEGG (http://www.genome.jp/kegg/), Human Metabolome Database (http://www.hmdb.ca/), SMPD (http://www.smpdb.ca/), and METLIN (http://metlin .scripps.edu/). Potential biological roles were evaluated by an enrichment analysis using MetaboAnalyst.

2.10. Statistical Analysis. All quantitative data analyses were performed using the SPSS 11.5 software package for Windows. Significance was determined using one-way analyses of variance (ANOVAs), followed by Student's $t$-test. The results were expressed as the mean \pm SD. $P$ values less than 0.05 were considered significant.

\section{Results and Discussion}

3.1. Potential Targets and Pathway Analysis. The 10 compounds that confirmed the absorption into the blood were imported into the database of PharmMapper to analyze the reverse docking. The results showed that 23 important potential protein targets were found, and these targets were put into the KEGG pathway annotation, the 15 pathways were discovered. Among these pathways there were 8 pathways related to inflammation and immunological stress. They are arachidonic acid metabolism, MAPK, adherens junction, focal adhesion, Fc epsilon RI, VEGF, B cell receptor signaling pathway, and T-cell receptor signaling pathway, respectively. The pathways associated with hormone metabolism are including androgen and estrogen metabolism, GnRH signaling pathway, and $\mathrm{ErbB}$ signaling pathway. Figure 2 showed the relationships between the ingredients, targets, and pathways.

3.2. Changes in Blood Indexes and Model Evaluation. Blood viscosity is the measure of how thin or thick the blood fluid is, and it reflects the blood flow and blood flow resistance. When blood is thick, blood flow is sluggish and there is an increased resistance, which tends to hinder normal energy metabolism and can lead to functional disorders in organs and tissues. In this experiment, whole blood viscosity and plasma viscosity indexes were determined for CCBS model rats. The whole blood viscosity (at high and low shear rates of $200 \mathrm{~s}^{-1}, 30 \mathrm{~s}^{-1}$, 


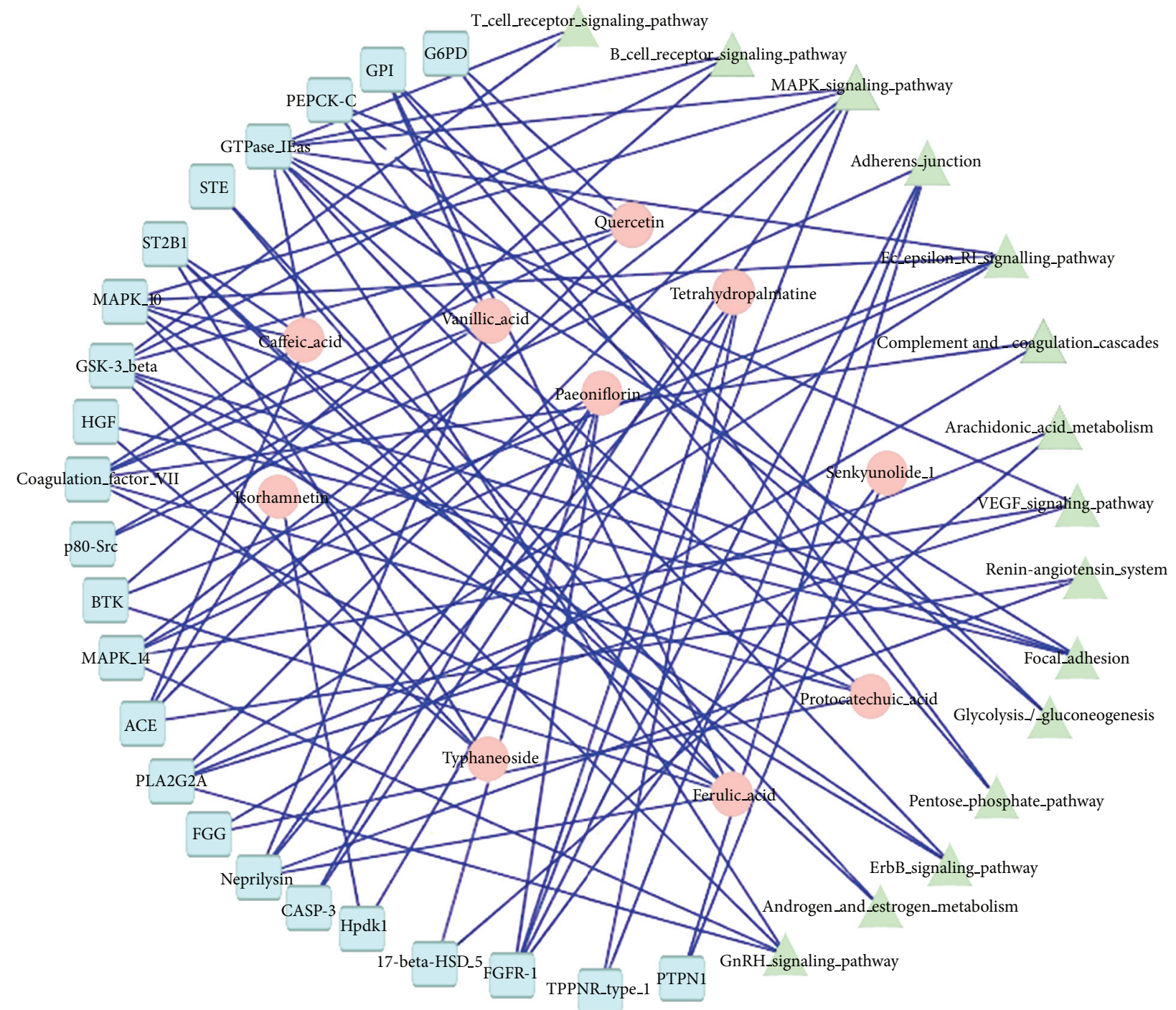

FIGURE 2: The relationships between the ingredients, targets, and pathways based on network biology.

$5 \mathrm{~s}^{-1}$, and $1 \mathrm{~s}^{-1}$ ) and plasma viscosity in model rat plasma were significantly increased $(P<0.05$ or 0.01 ) (Table 1 ), which suggests that the blood of these CCBS syndrome rats is in a viscous or stasis state. SFZYD significantly regulated whole blood viscosity at low shear rates $\left(5 \mathrm{~s}^{-1}\right.$ and $\left.1 \mathrm{~s}^{-1}\right)$ in model rats $(P<0.05)$ at 5.04 and $10.08 \mathrm{~g} \mathrm{~kg}^{-1}$ doses, respectively. At a dose of $10.08 \mathrm{~g} \mathrm{~kg}^{-1}$, SFZYD also significantly decreased whole blood viscosity at high shear rates $\left(30 s^{-1}\right.$ and $\left.200 s^{-1}\right)$ and plasma viscosity $(P<0.05$ or $P<0.01)$.

Coagulation is one important index for evaluating the state of blood stasis syndrome. The coagulation pathway comprises the complex interaction of many elements of the endothelium, coagulation factors, and platelets. Among these, thrombin plays a pivotal role in blood stasis syndrome. Thrombin acts as a multifunctional serine protease that is generated in response to vascular injury, and it catalyzes the proteolytic cleavage of the soluble plasma-protein fibrinogen to form insoluble fibrin, leading to clot formation. Thrombin also serves as a potent platelet agonist and amplifies its own generation via feedback activation of several steps in the coagulation cascade.

The PT and APTT assays were developed based on theories and the specific need for testing, without complete knowledge of all the proteins involved in coagulation. Our data showed that in model rats, the TT, PT, APTT, and FIB were remarkably decreased compared with those of normal rats. Furthermore, SFZYD prolonged TT and PT significantly $(P<0.05)$ in the model group (Table 2$)$. At a dosage of $10.08 \mathrm{~g} \mathrm{~kg}^{-1}$, SFZYD also showed activity toward APTT.

The determined data of 5-HT, NA, and $\beta$-EP were listed in Table 3. The results showed that the contents of 5-HT and $\mathrm{NA}$ in both brain tissue and plasma and of $\beta$-EP in plasma significantly increased in model rat $(P<0.05)$, while the content of $\beta$-EP in brain tissue was decreased in model rat $(P<0.05)$. After SFZYD treatment, the abnormal state was regulated remarkably $(P<0.05$ or $P<0.01)$. 
TABLE 1: Changes in the whole blood and plasma shear viscosity of model rats and SFZYD-treated rats $(n=6, \bar{x} \pm s)$.

\begin{tabular}{|c|c|c|c|c|c|}
\hline & \multicolumn{4}{|c|}{ Whole blood viscosity $(\eta \mathrm{b} / \mathrm{mPa} \cdot \mathrm{s})$} & \multirow{2}{*}{ plasma viscosity $(\eta \mathrm{b} / \mathrm{mPa} \cdot \mathrm{s})$} \\
\hline & $200\left(\mathrm{~s}^{-1}\right)$ & $30\left(\mathrm{~s}^{-1}\right)$ & $5\left(\mathrm{~s}^{-1}\right)$ & $1\left(\mathrm{~s}^{-1}\right)$ & \\
\hline Normal group & $4.01 \pm 0.13$ & $4.95 \pm 0.17$ & $7.58 \pm 0.46$ & $14.85 \pm 1.54$ & $2.45 \pm 0.16$ \\
\hline Model group & $4.24 \pm 0.10^{\Delta}$ & $5.46 \pm 0.19^{\Delta \Delta}$ & $8.99 \pm 0.59^{\Delta \Delta}$ & $19.19 \pm 2.01^{\Delta \Delta}$ & $3.08 \pm 0.60^{\Delta}$ \\
\hline SFZYD group $\left(5.4 \mathrm{~g} \mathrm{~kg}^{-1}\right)$ & $4.15 \pm 0.18$ & $5.20 \pm 0.23$ & $8.19 \pm 0.49^{*}$ & $16.61 \pm 1.48^{* *}$ & $2.84 \pm 0.32$ \\
\hline SFZYD group $\left(10.8 \mathrm{~g} \mathrm{~kg}^{-1}\right)$ & $4.00 \pm 0.11^{*}$ & $5.10 \pm 0.18^{*}$ & $7.89 \pm 0.53^{* *}$ & $15.86 \pm 1.63^{* *}$ & $2.67 \pm 0.21^{*}$ \\
\hline
\end{tabular}

${ }^{\Delta} P<0.05,{ }^{\Delta \Delta} P<0.01$ normal group versus model group; ${ }^{*} P<0.05$ model group versus SFZYD group.

TABLE 2: Changes in the plasma coagulation function of model rats and SFZYD-treated rats $(n=6, \bar{x} \pm s)$.

\begin{tabular}{lcccc}
\hline & TT $(\mathrm{s})$ & PT $(\mathrm{s})$ & APTT $(\mathrm{s})$ & FIB $(\mathrm{g} / \mathrm{L})$ \\
\hline Normal group & $31.5 \pm 3.9$ & $20.0 \pm 2.3$ & $47.2 \pm 4.7$ & $26.9 \pm 1.8$ \\
Model group & $22.7 \pm 2.0^{\Delta \Delta}$ & $14.4 \pm 1.7^{\Delta}$ & $34.8 \pm 3.1^{\Delta}$ & $18.4 \pm 2.9^{\Delta \Delta}$ \\
SFZYD group $\left(5.4 \mathrm{~g} \mathrm{~kg}^{-1}\right)$ & $28.2 \pm 2.3^{*}$ & $19.9 \pm 1.9^{*}$ & $36.6 \pm 3.6$ & $20.9 \pm 2.3$ \\
SFZYD group $\left(10.8 \mathrm{~g} \mathrm{~kg}^{-1}\right)$ & $30.9 \pm 3.3^{* *}$ & $20.9 \pm 1.2^{* *}$ & $38.9 \pm 2.8^{*}$ & $21.4 \pm 2.5$ \\
\hline
\end{tabular}

${ }^{\Delta} P<0.05,{ }^{\Delta \Delta} P<0.01$ normal group versus model group; ${ }^{*} P<0.05$ model group versus SFZYD group.

Moreover, we evaluated the model by analyzing metabolic profiling changes based on urine metabolomic data. The base peak intensity (BPI) chromatograms of the urine samples collected in positive ion mode during the eight days of animal model preparation are shown in (see Figure S1 Supplementary Material available online at http://dx.doi.org/10.1155/2013/901943). The results revealed that during the first three days of animal model preparation, there was no obvious departure of urine metabolic profiling, as determined by PCA, while pronounced changes were observed after 5 and 7 days of preparation, especially on the eighth day after the injection of hypodermic epinephrine, when there was a remarkable change in the metabolic profile (see Figure S2).

3.3. Metabolic Profiling Analysis. Typical base peak intensity (BPI) chromatograms in positive and negative ion modes of plasma and urine samples collected from normal and model rats are shown in Figure S3. The unsupervised PCA model was used to separate the plasma and urine samples into two blocks, respectively, between the normal group and the model group in positive and negative ion modes (Figures 3(a), 3(b), 3(c), 3(d), 4(a), 4(b), 4(c), and 4(d)). The supervised OPLS-DA, which could improve biomarker discovery and separate the samples into two blocks, was applied to obtain better discrimination between the two groups. The OPLS-DA score plot analysis of the chromatographic data identified the plasma and urine samples of the normal group and the model group based on the differences in their metabolic profiles, which suggested that the metabolic profiles were significantly changed as a result of CCBS syndrome (Figures 5(a), 5(b), $5(\mathrm{c})$, and $5(\mathrm{~d}))$. The recognition of a different pattern indicates that the endogenous metabolites have changed in CCBS syndrome model rats.

OPLS-DA distinguished normal rat and model rat cohorts with $100 \%$ sensitivity and no less than $95 \%$ specificity using a leave-one-out algorithm. The $R^{2} Y$ of this PLS-DA model was 0.926 and 0.917 (plasma samples) and 0.947 and 0.922 (urine samples) in the positive and negative ion modes, respectively. The $Q^{2}$ of the model was 0.849 and 0.853 (plasma samples) and 0.823 and 0.837 (urine samples), respectively. These results indicate that the OPLS-DA models were reliable.

3.4. Metabolites Identification. The ions furthest away from the origin contribute significantly to the clustering of the two groups, and they may be regarded as the potential biomarkers in model rats. Q-TOF was used to determine the precise molecular mass of these compounds within measurement errors $(<5 \mathrm{ppm})$. The potential elemental composition, degree of unsaturation, and fractional isotope abundance of the compounds were obtained. The presumed molecular mass was searched in the METLIN database, Chemspider, Human Metabolome Database, and other databases to identify the possible chemical constitutions, and MS/MS data were used to determine the possible structures of these ions.

Potential markers were extracted from S-plots and constructed following the OPLS analysis. Then, markers were chosen on the basis of their contribution to the variation and correlation within the data set. In the plasma, ten endogenous metabolites that contributed to the separation of the model rats and the normal rats were identified by comparing their molecular ion information and the corresponding fragments of product ions with authentic standards (Table 4). To illustrate the identification of metabolites, we will describe the ion at $t_{R}=15.97 \mathrm{~min}(\mathrm{~m} / z 496.3450)$ as an example. This ion may contain an odd number of nitrogen atoms because its precise molecular weight is 496.3383 , and its molecular formula was speculated to be $\mathrm{C}_{24} \mathrm{H}_{50} \mathrm{NO}_{7} \mathrm{P}$, based on the analysis of its elemental composition and fractional isotope abundance. In the positive ion spectrum, the main fragment ions that were analyzed via MS/MS screening were observed at $m / z 478.3290,419.2279,313.1615,184.0786,125.9252$, and 104.1071, which could be the $[\mathrm{M}+\mathrm{H}]^{+}$due to the loss of $-\mathrm{H}_{2} \mathrm{O}$, 
TABLE 3: The contents of 5-HT, NA, and $\beta$-EP in plasma and brain tissue of model rats and treatment group $(n=6, \bar{x} \pm s)$.

\begin{tabular}{|c|c|c|c|c|c|c|}
\hline & \multicolumn{2}{|c|}{ 5-HT (ng/mL) } & \multicolumn{2}{|c|}{$\mathrm{NA}(\mathrm{ng} / \mathrm{mL})$} & \multicolumn{2}{|c|}{$\beta$-EP (ng/mL) } \\
\hline & Brain tissue & Plasma & Brain tissue & Plasma & Brain tissue & Plasma \\
\hline Normal group & $144.39 \pm 30.36$ & $467.71 \pm 86.01$ & $3.92 \pm 0.72$ & $15.23 \pm 2.66$ & $0.28 \pm 0.10$ & $2.57 \pm 0.45$ \\
\hline Model group & $259.38 \pm 74.48^{\#}$ & $549.9 \pm 90.1^{\#}$ & $4.8 \pm 1.3^{\#}$ & $18.5 \pm 1.9^{\#}$ & $0.15 \pm 0.08^{\#}$ & $3.47 \pm 1.68^{\#}$ \\
\hline SFZYD group $\left(5.4 \mathrm{~g} \mathrm{~kg}^{-1}\right)$ & $183.49 \pm 89.99^{*}$ & $492.79 \pm 72.14^{*}$ & $4.33 \pm 1.46$ & $16.95 \pm 5.11^{*}$ & $0.24 \pm 0.09^{*}$ & $2.38 \pm 1.42^{*}$ \\
\hline SFZYD group $\left(10.8 \mathrm{~g} \mathrm{~kg}^{-1}\right)$ & $153.56 \pm 76.32^{* *}$ & $458.24 \pm 81.56^{* *}$ & $3.85 \pm 1.45^{*}$ & $15.87 \pm 4.36^{* *}$ & $0.34 \pm 0.12^{*}$ & $2.56 \pm 1.89^{*}$ \\
\hline
\end{tabular}

5-HT: 5-hydroxytryptamine; NA: Noradrenaline; $\beta$-EP: $\beta$-endorphin. Data were expressed as Mean \pm SEM. Means between the normal group, model group, low-dose SFZYD-treated group, and high-dose SFZYD-treated group. Significant differences when compared with the model group ${ }^{*} P<0.05$, ${ }^{* *} P<0.01$ and compared with the normal group ${ }^{\#} P<0.05$.

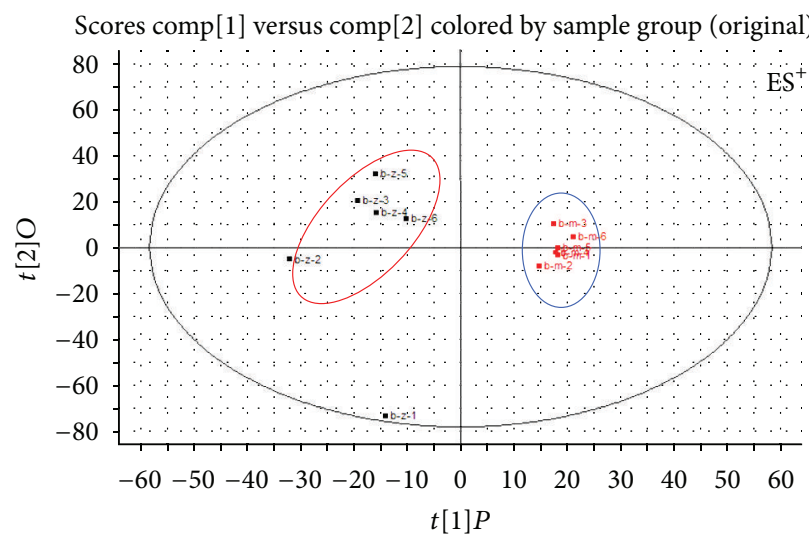

(a)

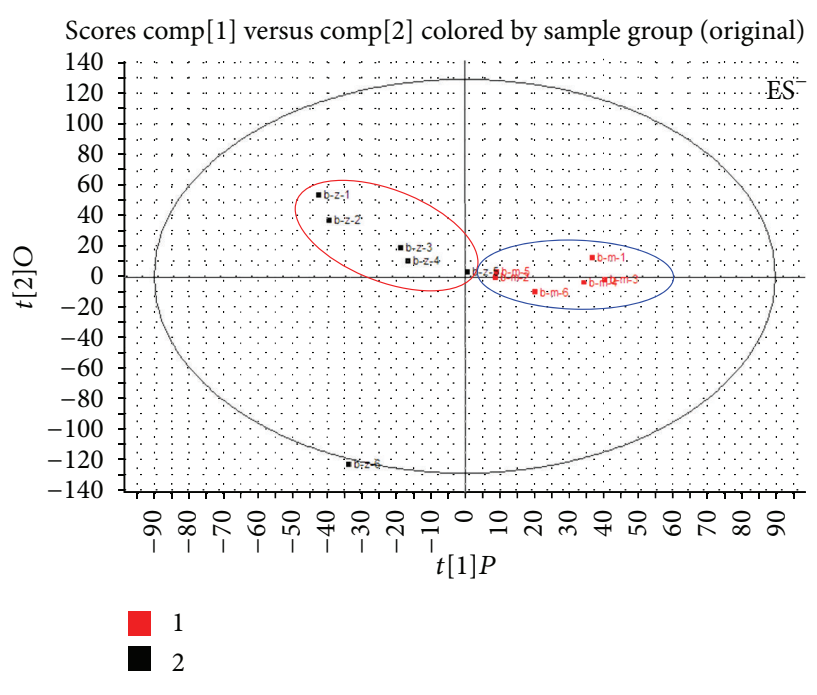

(c)

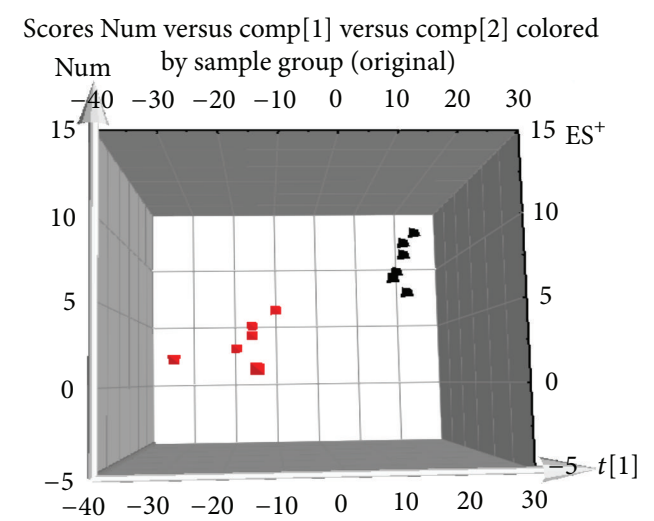

(b)

Scores Num versus comp[1] versus comp[2] colored
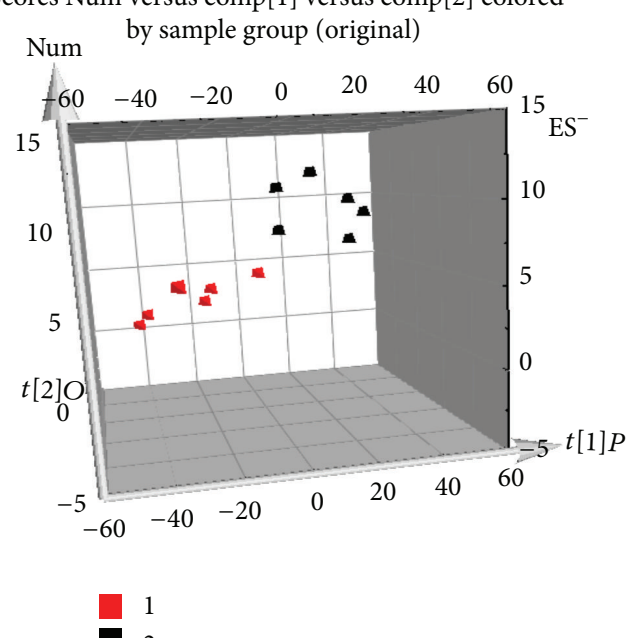

(c) (d)

FIGURE 3: PLS-DA model results for samples obtained from the plasma of the normal and model groups and analyzed in positive and negative ion modes $\left(\mathrm{ES}^{+}\right.$: (a) 2-D plot; (b) 3-D plot; $\mathrm{ES}^{-}$: (c) 2-D plot; (d) 3-D plot).

$-\mathrm{C}_{3} \mathrm{H}_{10} \mathrm{NO},-\mathrm{C}_{5} \mathrm{H}_{13} \mathrm{NO}_{4} \mathrm{P},-\mathrm{C}_{19} \mathrm{H}_{37} \mathrm{NO}_{2},-\mathrm{C}_{22} \mathrm{H}_{44} \mathrm{NO}_{3}$, and $-\mathrm{C}_{20} \mathrm{H}_{42} \mathrm{NO}_{4} \mathrm{P}$, respectively. Finally, to define its structure, we searched the HMDB database, and the metabolite was tentatively identified as lysophosphatidylcholine (16:0) [LPC $(16: 0)]$.

Compared with normal rats, four metabolites were upregulated $(P<0.05)$ in CCBS syndrome model rats, including 5dehydro-4-deoxy- $D$-glucarate, $5 \alpha$-tetrahydrocorticosterone, PC (13:0/0:0), and 17-phenyl trinor $\mathrm{PGF}_{2 \alpha}$ methyl ester.
Alternatively, six metabolites were significantly downregulated $(P<0.05)$ in CCBS syndrome model rats, including LysoPC (22:5 (7Z,10Z,13Z,16Z,19Z)), LysoPC (17:0), PC $(0: 0 / 18: 0)$, LysoPC $(18: 2(9 Z, 12 Z))$, LysoPC $(16: 0)$, and LysoPC (22: 6 (4Z, 7Z, 10Z, 13Z, 16Z, 19Z)) (Table 4).

In urine samples, the significant variables that were identified in positive and negative ion modes are summarized in Table 5. Ten endogenous metabolites were tentatively identified using the methods described above. The 


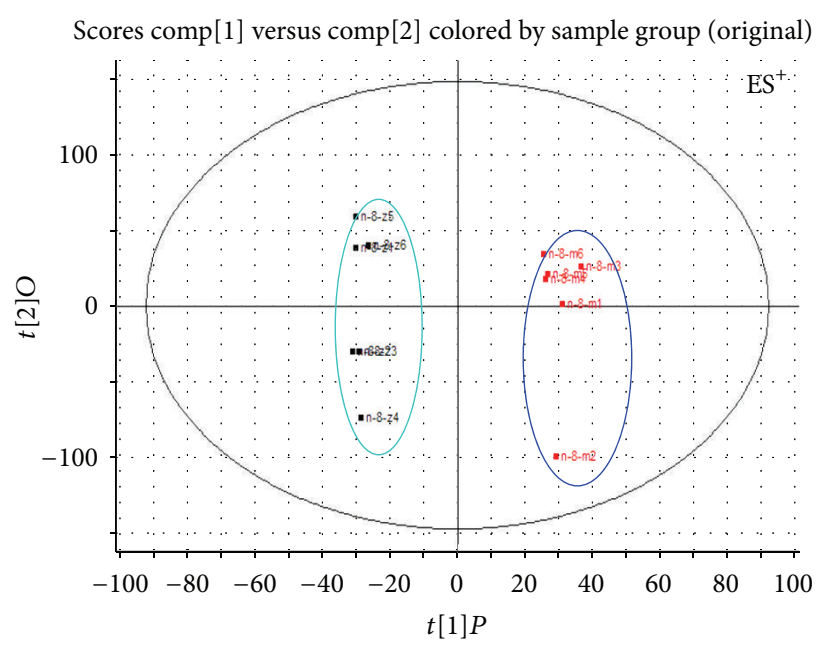

(a)

Scores comp[1] versus comp [2] colored by sample group (original)

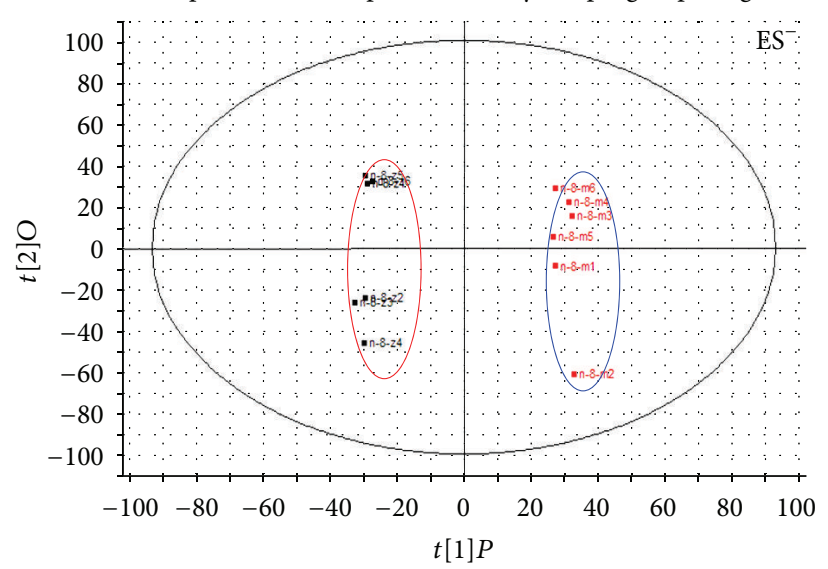

1
Scores comp[1] versus comp[2] versus comp [3] colored by sample group (original)

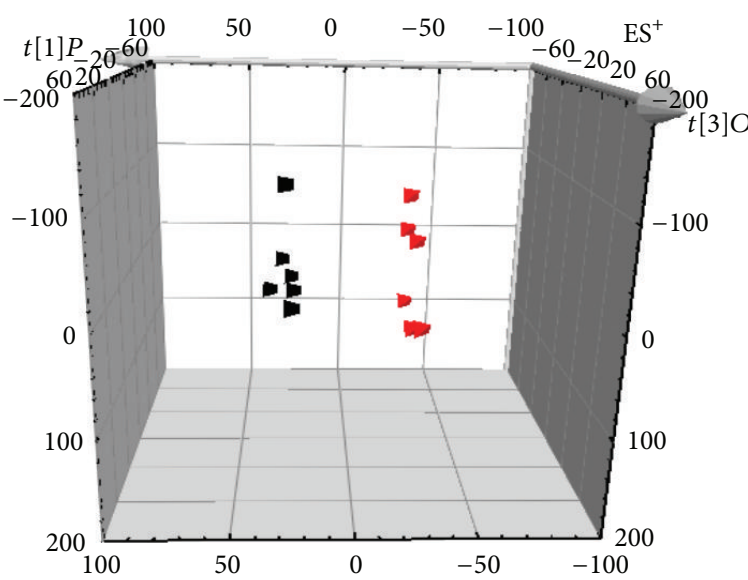

(b)

Scores comp[1] versus comp[2] versus comp [3] colored by sample group (original) $t[3] \mathrm{O}$

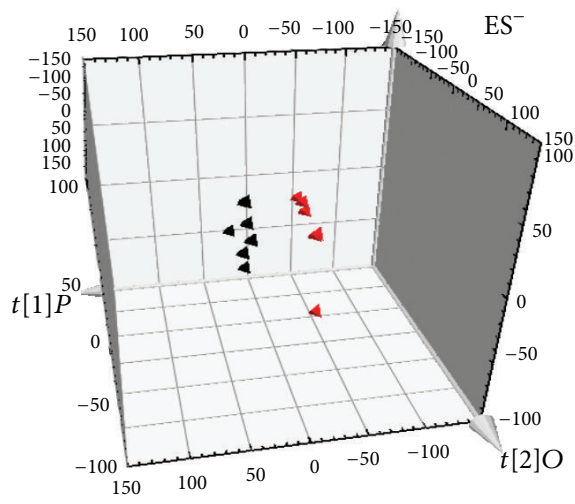

$\square \begin{aligned} & 1 \\ & 2\end{aligned}$

(c)

(d)

Figure 4: PLS-DA model results for the samples obtained from the urine of the normal and model groups and analyzed in positive and negative ion modes $\left(\mathrm{ES}^{+}\right.$: (a) 2-D plot; (b) 3-D plot; $\mathrm{ES}^{-}$: (c) 2-D plot; (d) 3-D plot).

metabolites of cholic acid, 3-methoxy-4-hydroxyphenylglycol sulfate, 5-dehydro-4-deoxy- $D$-glucarate, $5 \alpha$-tetrahydrocortisol, and 13,14-dihydro $\mathrm{PGF}_{2 \alpha}$ were significantly upregulated $(P<0.05)$ in CCBS syndrome model rats, whereas the metabolites of 2-phenylethanol glucuronide, hippuric acid, 6-hydroxy-5-methoxyindole glucuronide, 2,8-dihydroxyquinoline-beta-D-glucuronide, and normeperidinic acid glucuronide were significantly downregulated $(P<0.05)$. The fact that different metabolites were altered in the plasma and urine may denote the potential of these metabolites as targeted biomarkers that can be used to differentiate between the CCBS syndrome and normal states.

3.5. Metabolic Pathway and Function Analysis. Metabolite profiling is the analysis of a group of metabolites that are related to a specific metabolic pathway in biological states. More detailed analyses of the most relevant pathways and networks for CCBS were performed using Metaboanalyst, which is a free, web-based tool that combines the results of powerful pathway enrichment analysis with the conditions of the study. Metaboanalyst and directed graph use the high-quality KEGG (http://www.genome.jp/kegg/) pathway database as the backend knowledgebase. Consequently, the identification of potential targets using a metabolic pathway analysis (impact value $\geqslant 0.10$ ) with Metaboanalyst revealed that metabolites that were identified together are important for the host response to CCBS, and they are responsible for pentose and glucuronate interconversions and glycerophospholipid metabolism (see Figure S4). Distinct metabolic pathway analyses (impact value $\geqslant 0.10$ ) were performed to identify pathways related to CCBS.

3.6. Therapeutic Effects of SFZYD. To more clearly characterize the effects of SFZYD on CCBS model rats, 


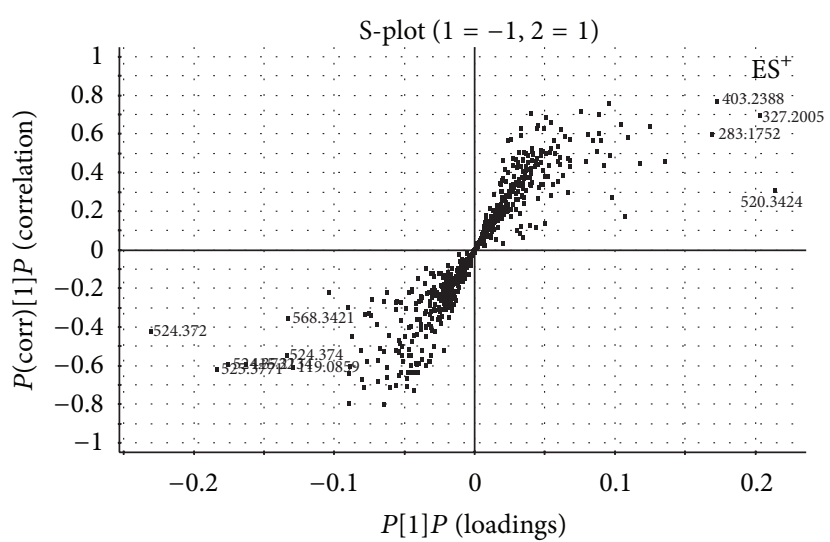

(a) Plasma

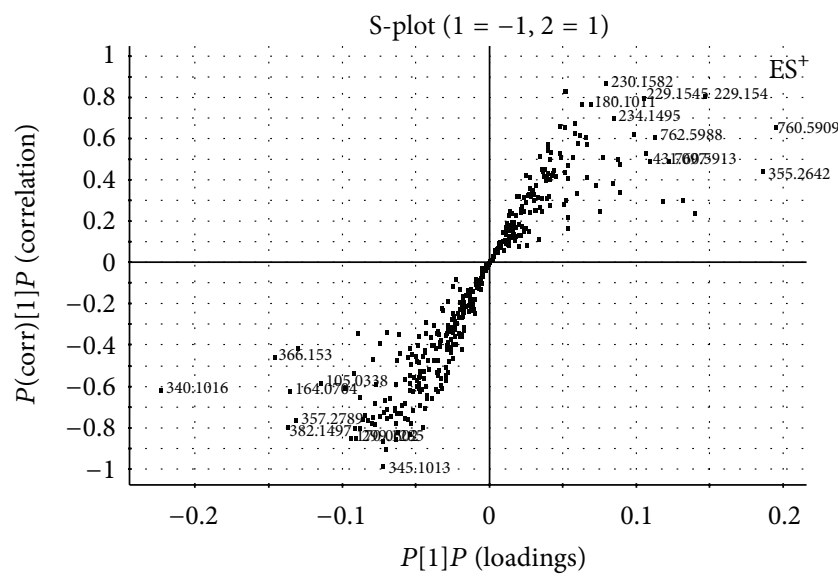

(c) Urine

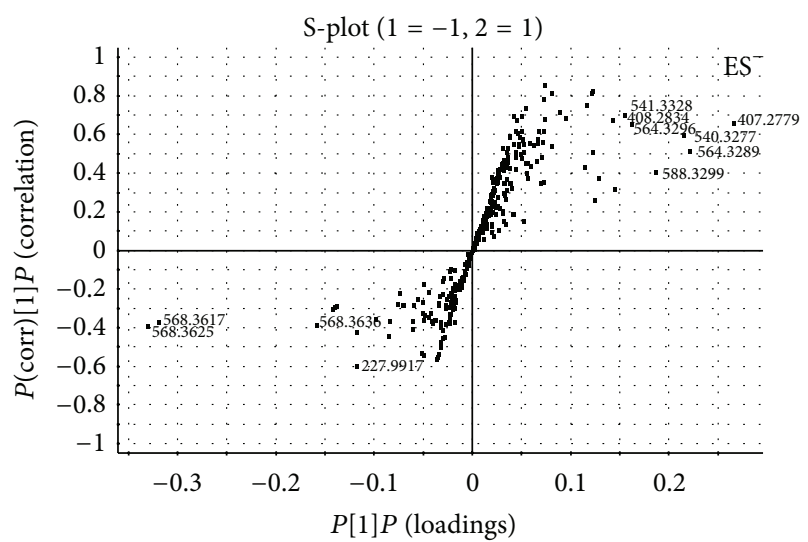

(b) Plasma

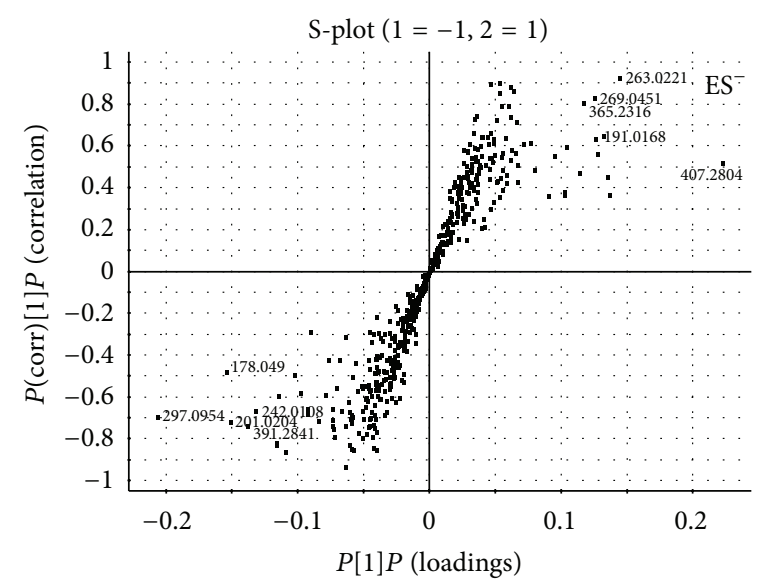

(d) Urine

FIGURE 5: S-plot of the OPLS-DA model for the plasma and urine samples from the normal versus model groups (plasma: (a) ES ${ }^{+}$mode; (b) $\mathrm{ESI}^{-}$mode; urine: (c) $\mathrm{ES}^{+}$mode; and (d) $\mathrm{ES}^{-}$mode).

TABLE 4: Identification of differentially expressed metabolites in the plasma that may account for the discrimination between normal and model rats.

\begin{tabular}{|c|c|c|c|c|c|c|}
\hline No. & Metabolites & Formula & Obsd. $[\mathrm{M}+\mathrm{H}]^{+} /[\mathrm{M}-\mathrm{H}]^{-}(m / z)$ & Content variance $^{\mathrm{c}}$ & $\mathrm{FC}^{\mathrm{d}}$ & $P^{\mathrm{e}}$ value \\
\hline 1 & LysoPC (22:5 (7Z, 10Z, 13Z, 16Z, 19Z)) & $\mathrm{C}_{30} \mathrm{H}_{52} \mathrm{NO}_{7} \mathrm{P}$ & $568.3436^{\mathrm{a}}$ & $\downarrow$ & -2.33 & 0.018 \\
\hline 2 & 5-Dehydro-4-deoxy-D-glucarate & $\mathrm{C}_{6} \mathrm{H}_{8} \mathrm{O}_{7}$ & $191.0197^{\mathrm{a}}$ & $\uparrow$ & 2.56 & 0.022 \\
\hline 3 & LysoPC (17:0) & $\mathrm{C}_{25} \mathrm{H}_{52} \mathrm{NO}_{7} \mathrm{P}$ & $508.3403^{\mathrm{a}}$ & $\downarrow$ & -1.42 & 0.030 \\
\hline 4 & $5 \alpha$-Tetrahydrocorticosterone & $\mathrm{C}_{21} \mathrm{H}_{34} \mathrm{O}_{4}$ & $349.2378^{\mathrm{a}}$ & $\uparrow$ & 2.56 & 0.035 \\
\hline 5 & $\mathrm{PC}(13: 0 / 0: 0)$ & $\mathrm{C}_{21} \mathrm{H}_{44} \mathrm{NO}_{7} \mathrm{P}$ & $452.2774^{\mathrm{a}}$ & $\uparrow$ & 1.25 & 0.021 \\
\hline 6 & 17-phenyl trinor PGF2 $\alpha$ methyl ester & $\mathrm{C}_{24} \mathrm{H}_{34} \mathrm{O}_{5}$ & $404.2438^{\mathrm{b}}$ & $\uparrow$ & 2.01 & 0.017 \\
\hline 7 & PC $(0: 0 / 18: 0)$ & $\mathrm{C}_{26} \mathrm{H}_{54} \mathrm{NO}_{7} \mathrm{P}$ & $524.3720^{\mathrm{b}}$ & $\downarrow$ & -1.35 & 0.042 \\
\hline 8 & LysoPC $(18: 2(9 \mathrm{Z}, 12 \mathrm{Z}))$ & $\mathrm{C}_{26} \mathrm{H}_{50} \mathrm{NO}_{7} \mathrm{P}$ & $520.3424^{\mathrm{b}}$ & $\downarrow$ & -1.56 & 0.030 \\
\hline 9 & LysoPC (16:0) & $\mathrm{C}_{24} \mathrm{H}_{50} \mathrm{NO}_{7} \mathrm{P}$ & $496.3450^{\mathrm{b}}$ & $\downarrow$ & -1.78 & 0.016 \\
\hline 10 & LysoPC (22:6 (4Z, 7Z, 10Z, 13Z, 16Z, 19Z)) & $\mathrm{C}_{30} \mathrm{H}_{50} \mathrm{NO}_{7} \mathrm{P}$ & $568.3421^{\mathrm{b}}$ & $\downarrow$ & -2.60 & 0.026 \\
\hline
\end{tabular}

${ }^{\mathrm{a}}$ Observed at $\mathrm{ES}^{-}$mode $[\mathrm{M}-\mathrm{H}]^{-} ;{ }^{\mathrm{b}}$ observed at $\mathrm{ES}^{+}$mode $[\mathrm{M}+\mathrm{H}]^{+}$.

${ }^{c} \uparrow$ : content increased; $\downarrow$ : content decreased.

${ }^{\mathrm{d}}$ Fold change was calculated as the ratio of the mean metabolite levels between two groups. A positive value of fold change indicates a relatively higher concentration of metabolites, while a negative value of fold change indicates a relatively lower concentration of metabolites in model rats as compared to normal rats.

${ }^{\mathrm{e}} P$ values were calculated from two-tailed Mann-Whitney $U$ test with a threshold of 0.05 . 


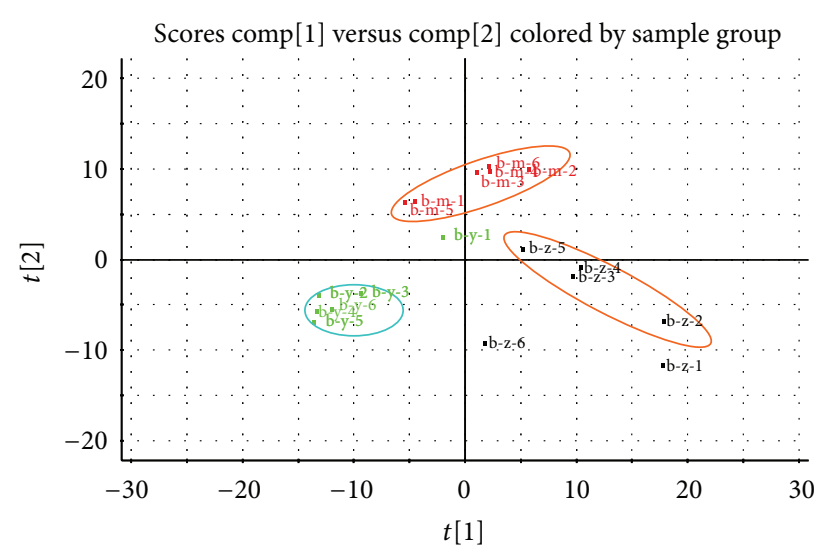

(a) Plasma

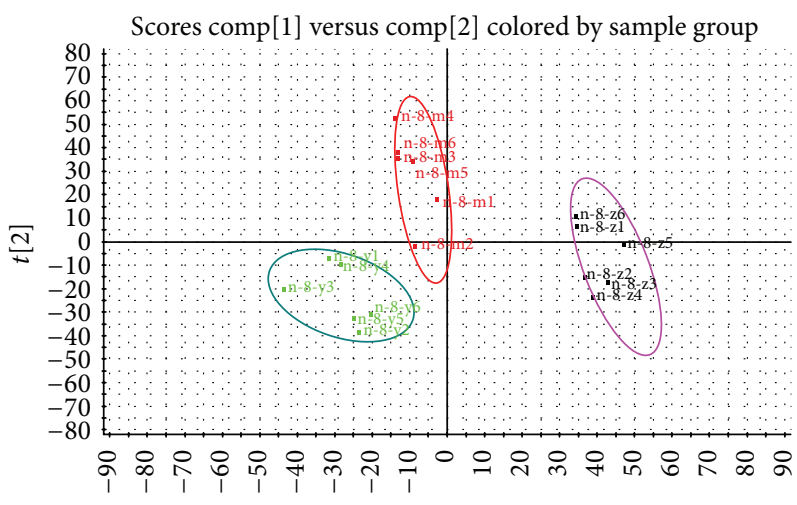

$t[1]$

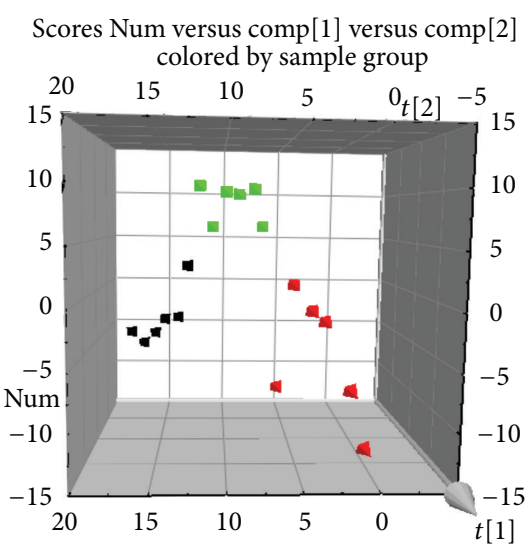

(b) Plasma

Scores comp[1] versus comp[2] versus

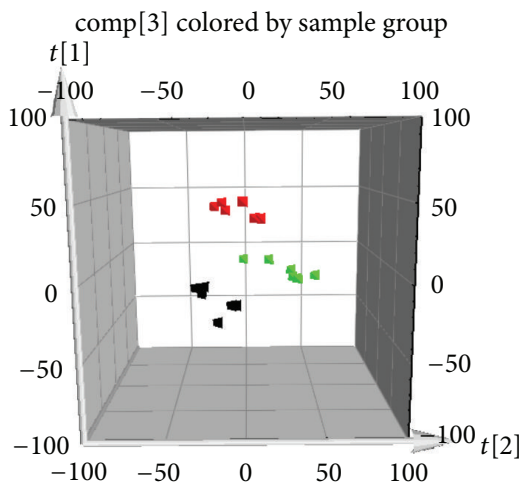

(c) Urine

(d) Urine

FIGURE 6: PLS-DA model results and loadings plots for the plasma and urine samples obtained from the normal, model, and treatment groups and analyzed in positive ion mode (Plasma: (a) 2-D plot and (b) 3-D plot; Urine: (c) 2-D plot and (d) 3-D plot).

TABLE 5: Identification of differentially expressed metabolites in the urine that may account for the discrimination between normal and model rats.

\begin{tabular}{|c|c|c|c|c|c|c|}
\hline No. & Metabolites & Formula & Obsd. $[\mathrm{M}+\mathrm{H}]^{+} /[\mathrm{M}-\mathrm{H}]^{-}(m / z)$ & Content variance $^{c}$ & $\mathrm{FC}^{\mathrm{d}}$ & $P^{\mathrm{e}}$ value \\
\hline 1 & Cholic acid & $\mathrm{C}_{24} \mathrm{H}_{40} \mathrm{O}_{5}$ & $407.2804^{\mathrm{a}}$ & $\uparrow$ & 1.56 & 0.035 \\
\hline 2 & 2-Phenylethanol glucuronide & $\mathrm{C}_{14} \mathrm{H}_{18} \mathrm{O}_{7}$ & $297.0954^{\mathrm{a}}$ & $\downarrow$ & -1.69 & 0.026 \\
\hline 3 & Hippuric acid & $\mathrm{C}_{9} \mathrm{H}_{9} \mathrm{NO}_{3}$ & $178.0491^{\mathrm{a}}$ & $\downarrow$ & -1.81 & 0.040 \\
\hline 4 & 3-Methoxy-4-hydroxyphenylglycol sulfate & $\mathrm{C}_{9} \mathrm{H}_{12} \mathrm{O}_{7} \mathrm{~S}$ & $263.0221^{\mathrm{c}}$ & $\uparrow$ & 2.53 & 0.025 \\
\hline 5 & 5-Dehydro-4-deoxy-D-glucarate & $\mathrm{C}_{6} \mathrm{H}_{8} \mathrm{O}_{7}$ & $191.0168^{\mathrm{a}}$ & $\uparrow$ & 2.40 & 0.016 \\
\hline 6 & $5 \alpha$-Tetrahydrocortisol & $\mathrm{C}_{21} \mathrm{H}_{34} \mathrm{O}_{5}$ & $365.2316^{\mathrm{a}}$ & $\uparrow$ & 3.70 & 0.023 \\
\hline 7 & 6-Hydroxy-5-methoxyindole glucuronide & $\mathrm{C}_{15} \mathrm{H}_{17} \mathrm{NO}_{8}$ & $340.1016^{\mathrm{b}}$ & $\downarrow$ & -3.79 & 0.012 \\
\hline 8 & 2,8-Dihydroxyquinoline-beta-D-glucuronide & $\mathrm{C}_{15} \mathrm{H}_{15} \mathrm{NO}_{8}$ & $338.0847^{\mathrm{b}}$ & $\downarrow$ & -2.42 & 0.028 \\
\hline 9 & Normeperidinic acid glucuronide & $\mathrm{C}_{18} \mathrm{H}_{23} \mathrm{NO}_{8}$ & $382.1497^{\mathrm{b}}$ & $\downarrow$ & -3.21 & 0.037 \\
\hline 10 & 13,14-dihydro PGF2 $\alpha$ & $\mathrm{C}_{20} \mathrm{H}_{36} \mathrm{O}_{5}$ & $357.2724^{\mathrm{b}}$ & $\uparrow$ & 2.01 & 0.028 \\
\hline
\end{tabular}

${ }^{\mathrm{a}}$ Observed at $\mathrm{ES}^{-}$mode $[\mathrm{M}-\mathrm{H}]^{-} ;{ }^{\mathrm{b}}$ observed at $\mathrm{ES}^{+}$mode $[\mathrm{M}+\mathrm{H}]^{+}$.

${ }^{c} \uparrow$ : content increased; $\downarrow$ : content decreased.

${ }^{d}$ Fold change was calculated as the ratio of the mean metabolite levels between two groups. A positive value of fold change indicates a relatively higher concentration of metabolites while a negative value of fold change indicates a relatively lower concentration of metabolites in model rats as compared to normal rats.

${ }^{\mathrm{e}} P$ values were calculated from two-tailed Mann-Whitney $U$ test with a threshold of 0.05 . 


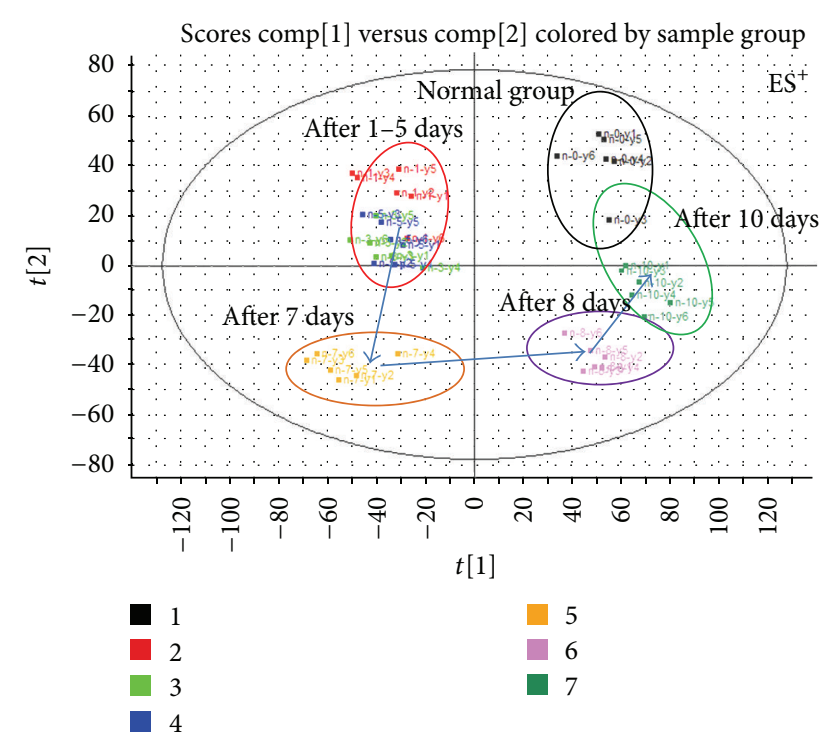

(a)

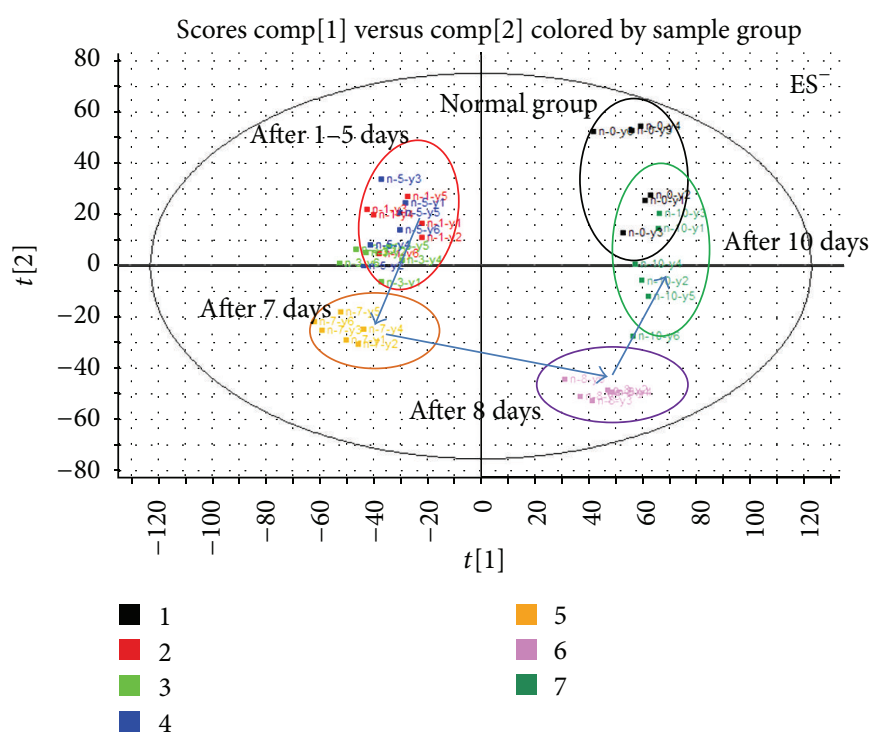

(b)

FIGURE 7: Urine metabolic profile changes after days $0,1,3,5,7,8$, and 10 of SFZYD treatment, as analyzed in positive and negative ion modes ((a) $\mathrm{ES}^{+}$mode; (b) $\mathrm{ES}^{-}$mode) [23].

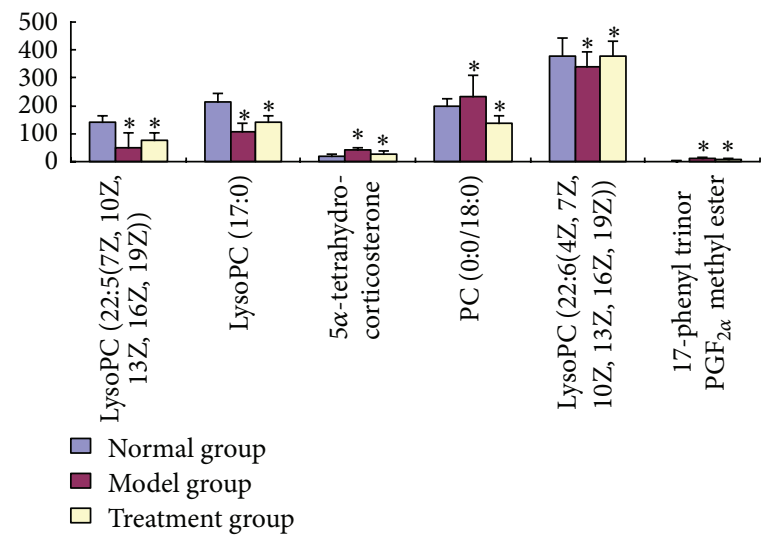

(a) Plasma

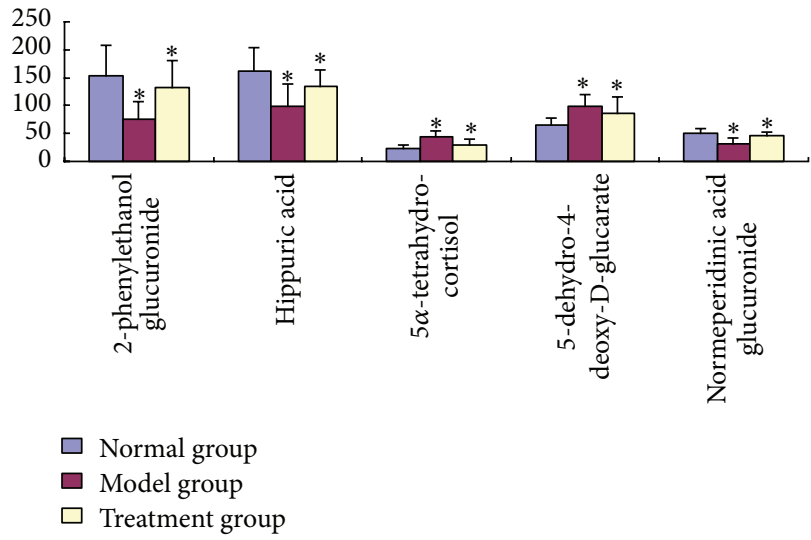

(b) Urine

FIGURE 8: Changes in the relative quantities of targeted metabolites in the plasma and urine, identified in different groups. A two-tailed, parametric $t$-test was used to determine the significance of the changes for each metabolite, in relative quantities. Bars represent the mean relative metabolite concentration and standard deviations. ${ }^{*} P<0.05$.

a PCA analysis was carried out to determine the changes before and after SFZYD treatment. The results revealed variations between the plasma and urine metabolic profiles of the model group, normal group, and SFZYD group (Figures 6(a), 6(b), 6(c), and 6(d)). To better understand the time-dependent effect of SFZYD, a PCA model was constructed to analyze all the data acquired from the normal group, predose group and treatment group at days 1, 3, 5, 7 , and 10 in both positive and negative ion modes (Figures $7(\mathrm{a})$ and $7(\mathrm{~b})$ ). The spot observed in the treatment group at days 1-5 is close to that of the model group, indicating that the cold coagulation and blood stasis syndrome state is dominant. The spots observed in the treatment group at day 7 clustered near the center of the plot with a shift back toward the normal group, which might be an indication of the accumulated effect of SFZYD. The spot observed in the treatment group at days 8 and 10 ultimately approached the normal state, suggesting that SFZYD treatment had a positive therapeutic effect on the rats. Furthermore, the relative concentrations of 6 endogenous metabolites in the plasma and 5 endogenous metabolites in the urine were significantly affected by SFZYD $(P<0.05)$. All of these metabolites returned to normal levels after SFZYD treatment (Figures $8(\mathrm{a})$ and $8(\mathrm{~b})$ ). Thus, the efficient regulation of these potential biomarkers may account for the effects of SFZYD in model rats.

The prediction and identification of molecular markers (targets) and metabolic pathways have the potential to improve the diagnosis, prognosis, and therapy for ZHENG or diseases [27-30]. We utilized reverse docking method to 
predict the biology targets and pathway annotation. Furthermore a metabolomic approach to analyze the changes in the plasma and urine samples of CCBS syndrome model rats, normal rats, and SFZYD-treated rats was applied. We identified 20 endogenous metabolites (10 in the plasma and 10 in the urine) that were upregulated or downregulated $(P<$ 0.05 or 0.01 ) in CCBS syndrome, including LysoPCs and glucuronide metabolites. Furthermore, 11 potential biomarkers (6 metabolites in the plasma and 5 metabolites the in urine) were regulated by SFZYD.

Studies using targeted metabolite analyses have already shown that alterations in critical CCBS syndrome metabolic pathways, such as glycerophospholipid metabolism (impact value 0.24 ) and pentose and glucuronate interconversions (impact value 0.27 ), are strongly associated with the development of CCBS syndrome. Phospholipid metabolism and glycometabolism were disturbed in the plasma and urine of CCBS rats, respectively. In urine, the levels of the metabolites of 2-phenylethanol glucuronide, hippuric acid, and normeperidinic acid glucuronide, which are all related to glycometabolism, were significantly decreased in model rats. The low level of glucuronide metabolites implies that energy metabolism decreased and the energy metabolism pathway increased, resulting in abnormal pentose and glucuronate interconversions. Previous studies have also reported that cold exposure increased energy expenditure by activating specific sympathetic pathways [31]. These results agreed well with the SFZYD bioactive ingredients prediction of network targets related to inflammation and immunological stress, hormone metabolism, glycometabolism, and coagulation cascade system.

Ephedrine is known to raise blood pressure, heart rate, and energy expenditure and to increase the levels of multiple circulating metabolites, including glucose, insulin, and thyroid hormones [31]. In this paper, cold and ephedrine mutually induced CCBS syndrome in model rats, and the level of phosphatidylcholine in the plasma decreased. Among the potential markers, $5 \alpha$-tetrahydrocorticosterone and 17 phenyl trinor PGF2 $\alpha$ methyl ester levels increased in the plasma of the model rats. $5 \alpha$-Tetrahydrocorticosterone is a corticosteroid hormone, and the hypothalamic-pituitaryadrenal axis was activated in the CCBS model rats induced with cold and epinephrine. The excretion of corticotropinreleasing factor (CRF) by the hypothalamus and pituitarium leads to the release of adrenocorticotropic hormone, which acts on the adrenal cortex to promote the secretion of corticosteroid hormones [32]. Therefore, the combination of cold and ephedrine could change the metabolism of hormones in CCBS syndrome model rats. Moreover, the levels of LysoPC metabolites were decreased in the plasma of model rats.

It was reported that primary dysmenorrhea patients with CCBS syndrome have high levels of prostaglandin growth factor 2 (PGF2) in their menstrual fluid [33], and PGF2 stimulates myometrial contractions and ischemia and sensitizes nerve endings. In this paper, CCBS syndrome model rats had high levels of 17-phenyl trinor $\mathrm{PGF}_{2 \alpha}$ methyl ester, and phospholipid metabolism was disturbed, likely due to the inflammatory response. Future research will focus on the discovery of additional biomarkers using metabolomics platforms and the validation of explorative biomarkers.

In addition, 11 specific metabolites regulated by SFZYD were identified, including $5 \alpha$-tetrahydrocortisol, 5-dehydro4-deoxy-D-glucarate, 2-phenylethanol glucuronide, hippuric acid, and normeperidinic acid glucuronide in the urine and $5 \alpha$-tetrahydrocorticosterone, PC $(0: 0 / 18: 0)$, 17-phenyl trinor $\mathrm{PGF}_{2 \alpha}$ methyl ester, LysoPC (22:6(4Z, 7Z, 10Z, 13Z, 16Z, 19Z)), LysoPC (17:0), and LysoPC (22:6 (4Z, 7Z, 10Z, $13 Z, 16 Z, 19 Z))$ in the plasma. These biomarkers suggest that the pathogenesis of CCBS syndrome is closely related to glycometabolism and phospholipid metabolism. Based on these findings, further studies would be performed to validate the predicted targets and the changed metabolites and to elucidate the mechanisms underlying these alterations.

Our results also showed that SFZYD improved the status of hemorheology and blood viscosity, and it regulated the coagulation function of TT and APTT. These data imply that the state of CCBS syndrome is closely associated with blood coagulation function. This research also verified that ephedrine-induced platelet aggregation, gluconeogenesis, ischemia, and $\mathrm{Ca}^{2+}$ influx in vascular endothelial cells are mediated by CNGA2 channels [34-37]. So, the systems biology and network targets prediction are one of the most important trends for the development of traditional Chinese medicine [38-40].

\section{Conclusions}

In summary, the reverse docking method and metabolomicsbased study provide a powerful approach to evaluate the effects of Chinese herbs and discover potential biomarkers (targets) via the prediction of biological targets and analysis of global changes in an individual's metabolic profile. Here, for the first time, we performed a comprehensive analysis of the network targets, pathways induced by SFZYD bioactive ingredients, and metabolic patterns of CCBS syndrome. Our findings suggest that the proposed approach would be helpful for establishing a suitable model to reasonably evaluate CCBS syndrome, explore its pathological mechanisms, and clarify the mechanisms of action of SFZYD.

\section{Abbreviations \\ PD: $\quad$ Primary dysmenorrhea \\ SFZYD: Shaofu Zhuyu decoction \\ MS/MS: Tandem mass spectrometry \\ OPLS: Orthogonal partial least squares \\ PCA: Principal components analysis \\ PLS-DA: Partial least-squares discriminant analysis \\ RT: Retention time \\ TOFMS: Time-of-flight mass spectrometry \\ UPLC: Ultraperformance liquid chromatography.}

\section{Acknowledgments}

This work was supported by the Key Research Project in Basic Science of Jiangsu College and University (nos. 06KJA36022 
and 11KJA360002) and the National Natural Science Foundation of China (nos. 30973885, 81373889 and 81102898). This work was also supported by the Construction Project for Jiangsu Key Laboratory for High Technology Research of TCM Formulae (BM2010576; BK2010561), the Construction Project for Jiangsu Engineering Center of Innovative Drug from Blood-conditioning TCM Formulae, and a project funded by the Priority Academic Program Development of Jiangsu Higher Education Institutions (ysxk-2010).

\section{References}

[1] T. Xue and R. Roy, "Studying traditional Chinese medicine," Science, vol. 300, no. 5620, pp. 740-741, 2003.

[2] S. Li, Z. Q. Zhang, L. J. Wu, X. G. Zhang, Y. D. Li, and Y. Y. Wang, "Understanding ZHENG in traditional Chinese medicine in the context of neuro-endocrine-immune network," IET Systems Biology, vol. 1, no. 1, pp. 51-60, 2007.

[3] S. B. Su, A. P. Lu, S. Li, and W. Jia, "Evidence-based ZHENG: a traditional chinese medicine syndrome," Evidence-Based Complementary and Alternative Medicine, vol. 2012, Article ID 246538, 2 pages, 2012.

[4] F. Liao, "Herbs of activating blood circulation to remove blood stasis," Clinical Hemorheology and Microcirculation, vol. 23, no. 2-4, pp. 127-131, 2000.

[5] X.-J. Ma, H.-J. Yin, and K.-J. Chen, "Investigation of gene expression profiles in patients with blood stasis syndrome," Journal of Chinese Integrative Medicine, vol. 6, no. 4, pp. 355360, 2008.

[6] S. L. Su, J. A. Duan, T. J. Wang, and Y. P. Tang, "Evaluating the effects of Shaofu Zhuyu Decoction on hemorheology and ovarian function in rat model of Han-Ning blood stasis," Chinese Journal of Experimental Traditional Medicine Formula, vol. 14, no. 12, pp. 14-34, 2008 (Chinese).

[7] J. Zhang and C. L. Sun, "Application in gynecology diseases of Shaofu Zhuyu decoction," Chinese Journal of Natural Medicine, vol. 4, no. 2, pp. 109-112, 2002 (Chinese).

[8] M. Fan, "Clinical observation of Shaofu Zhuyu decotiong for treating primary dysmenorrhea of cold coagulation and blood stasis," Beijing Journal of Traditional Chinese Medicine, vol. 30, no. 6, pp. 455-456, 2011 (Chinese).

[9] Q. Q. Cheng and Y. Zhu, "Clinical study on primary dysmenorrheal of blood-stasis of cold-coagulation syndrome treated with modified Shaofu Zhuyu decoction," Journal of Chinese Medicine, vol. 26, no. 10, pp. 1249-1250 (Chinese).

[10] J. K. Nicholson and I. D. Wilson, "Understanding "global" systems biology: metabonomics and the continuum of metabolism," Nature Reviews Drug Discovery, vol. 2, no. 8, pp. 668-676, 2003.

[11] C.-X. Liu, "Metabonomics in medical and pharmaceutical research," Acta Academiae Medicinae Sinicae, vol. 29, no. 6, pp. 712-718, 2007.

[12] X. Wang, B. Yang, A. Zhang, H. Sun, and G. Yan, "Potential drug targets on insomnia and intervention effects of Jujuboside A through metabolic pathway analysis as revealed by UPLC/ESISYNAPT-HDMS coupled with pattern recognition approach," Journal of Proteomics, vol. 75, no. 4, pp. 1411-1427, 2012.

[13] Y. H. Lu, H. P. Hao, G. J. Wang, and X. H. Chen, "Metabolomics approach to the biochemical differentiation of traditional Chinese medicine syndrome types of hypertension," Chinese
Journal of Clinical Pharmacology Therapeutics, vol. 12, no. 10, pp. 1144-1150, 2007 (Chinese).

[14] P. Gong, N. Cui, L. Wu et al., "Chemicalome and metabolome matching approach to elucidating biological metabolic networks of complex mixtures," Analytical Chemistry, vol. 84, no. 6, pp. 2995-3002, 2012.

[15] X. Wang, H. Wang, A. Zhang et al., "Metabolomics study on the toxicity of aconite root and its processed products using ultraperformance liquid-chromatography/electrosprayionization synapt high-definition mass spectrometry coupled with pattern recognition approach and ingenuity pathways analysis," Journal of Proteome Research, vol. 11, no. 2, pp. 12841301, 2012.

[16] H. Y. Zhao, J. Li, X. J. He et al., "The protective effect of Yi Shen Juan Bi Pill in arthritic rats with castration-induced kidney deficiency," Evidence-Based Complementary and Alternative Medicine, vol. 2012, Article ID 102641, 8 pages, 2012.

[17] J. Li, C. Lu, M. Jiang et al., "Traditional Chinese Medicinebased network pharmacology could lead to new multicompound drug discovery," Evidence-Based Complementary and Alternative Medicine, vol. 2012, Article ID 149762, 11 pages, 2012.

[18] S. Li and B. Zhang, "Traditional Chinese medicine network pharmacology: theory, methodology and application," Chinese Journal of Natural Medicines, vol. 11, no. 2, pp. 110-120, 2013.

[19] B. Zhang, X. Wang, and S. Li, "An integrative platform of TCM network pharmacology and its application on an herbal formula, Qing-Luo-Yin," Evidence-Based Complementary and Alternative Medicine, vol. 2013, Article ID 456747, 12 pages, 2013.

[20] S. Li, B. Zhang, and N. Zhang, "Network target for screening synergistic drug combinations with application to traditional Chinese medicine," BMC Systems Biology, vol. 5, supplement 1, article S10, 2011.

[21] S. Li, B. Zhang, D. Jiang, Y. Wei, and N. Zhang, "Herb network construction and co-module analysis for uncovering the combination rule of traditional Chinese herbal formulae," BMC Bioinformatics, vol. 11, supplement 11, article S6, 2010.

[22] S. Li, "Network systems underlying traditional Chinese medicine syndrome and herb formula," Current Bioinformatics, vol. 4, no. 3, pp. 188-196, 2009.

[23] S. L. Su, W. X. Cui, J. A. Duan et al., "UHPLC-MS simultaneous determination and pharmacokinetic study of three aromatic acids and one monoterpene in rat plasma after oral administration of Shaofu Zhuyu Decoction," The American Journal of Chinese Medicine, vol. 41, no. 3, pp. 697-715, 2013.

[24] S. Su, J. Guo, J.-A. Duan et al., "Ultra-performance liquid chromatography-tandem mass spectrometry analysis of the bioactive components and their metabolites of Shaofu Zhuyu decoction active extract in rat plasma," Journal of Chromatography B, vol. 878, no. 3-4, pp. 355-362, 2010.

[25] H.-X. Li, S.-Y. Han, X.-W. Wang et al., "Effect of the carthamins yellow from Carthamus tinctorius L. on hemorheological disorders of blood stasis in rats," Food and Chemical Toxicology, vol. 47, no. 8, pp. 1797-1802, 2009.

[26] E. M. Lenz and I. D. Wilson, "Analytical strategies in metabonomics," Journal of Proteome Research, vol. 6, no. 2, pp. 443-458, 2007.

[27] G. S. Catchpole, M. Beckmann, D. P. Enot et al., "Hierarchical metabolomics demonstrates substantial compositional similarity between genetically modified and conventional potato crops," Proceedings of the National Academy of Sciences of the United States of America, vol. 102, no. 40, pp. 14458-14462, 2005. 
[28] J.-Y. Liu, N. Li, J. Yang et al., "Metabolic profiling of murine plasma reveals an unexpected biomarker in rofecoxib-mediated cardiovascular events," Proceedings of the National Academy of Sciences of the United States of America, vol. 107, no. 39, pp. 17017-17022, 2010.

[29] K. Suhre, S. Y. Shin, A. K. Petersen et al., "Human metabolic individuality in biomedical and pharmaceutical research," Nature, vol. 477, no. 7326, pp. 54-60, 2011.

[30] D. D. Andersona, C. M. Quintero, and P. J. Stovera, "Identification of a de novo thymidylate biosynthesis pathway in mammalian mitochondria," Proceedings of the National Academy of Sciences of the United States of America, vol. 108, no. 37, pp. 15163-15168, 2011.

[31] A. M. Cypess, Y. C. Chen, C. Sze et al., "Cold but not sympathomimetics activates human brown adipose tissue in vivo," Proceeding of the National Academy of Science of the United States of America, vol. 109, no. 25, pp. 10001-10005, 2012.

[32] L. Arborelius, M. J. Owens, P. M. Plotsky, and C. B. Nemeroff, "The role of corticotropin-releasing factor in depression and anxiety disorders," Journal of Endocrinology, vol. 160, no. 1, pp. 1-12, 1999.

[33] W. Y. Chan and M. Y. Dawood, "Prostaglandin levels in menstrual fluid of nondysmenorrheic and of dysmenorrheic subjects with and without oral contraceptive or ibuprofen therapy," Advances in prostaglandin and thromboxane research, vol. 8, pp. 1443-1447, 1980.

[34] A. Spalding, H. Vaitkevicius, S. Dill, S. MacKenzie, A. Schmaier, and W. Lockette, "Mechanism of epinephrine-induced platelet aggregation," Hypertension, vol. 31, no. 2, pp. 603-607, 1998.

[35] K. Kim, C. C. Sung, A. Cova, I. S. Jang, and S. C. Park, "Alterations of epinephrine-induced gluconeogenesis in aging," Experimental and Molecular Medicine, vol. 41, no. 5, pp. 334340, 2009.

[36] B. H. Sung, M. F. Wilson, C. Robinson, U. Thadani, and W. R. Lovallo, "Mechanisms of myocardial ischemia induced by epinephrine: comparison with exercise-induced ischemia," Psychosomatic Medicine, vol. 50, no. 4, pp. 381-393, 1988.

[37] B. Shen, K.-T. Cheng, Y.-K. Leung et al., "Epinephrine-induced $\mathrm{Ca}^{2+}$ influx in vascular endothelial cells is mediated by CNGA2 channels," Journal of Molecular and Cellular Cardiology, vol. 45, no. 3, pp. 437-445, 2008.

[38] X. Wang, B. Yang, H. Sun, and A. Zhang, "Pattern recognition approaches and computational systems tools for ultra performance liquid chromatography-mass spectrometry-based comprehensive metabolomic profiling and pathways analysis of biological data sets," Analytical Chemistry, vol. 84, no. 1, pp. 428-439, 2012.

[39] S. L. Su, J. A. Duan, P. J. Wang et al., "Metabolomic study of biochemical changes in the plasma and urine of primary dysmenorrhea patients using UPLC-MS coupled with a pattern recognition approach," Journal of Proteome Research, vol. 12, no. 2, pp. 852-865, 2013.

[40] T. Ma, C. Tan, H. Zhang, M. Wang, W. Ding, and S. Li, "Bridging the gap between traditional Chinese medicine and systems biology: the connection of cold syndrome and NEI network," Molecular BioSystems, vol. 6, no. 4, pp. 613-619, 2010. 


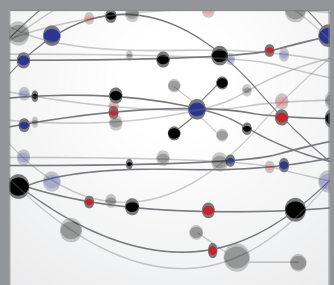

The Scientific World Journal
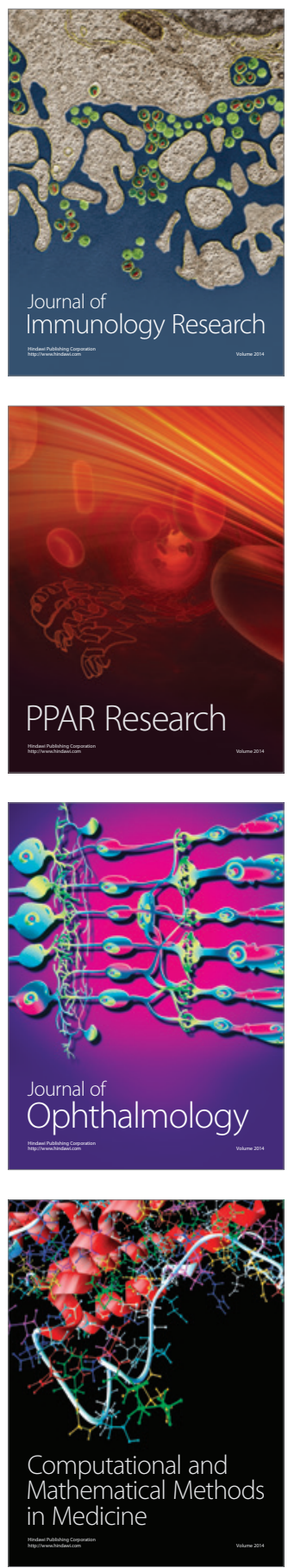

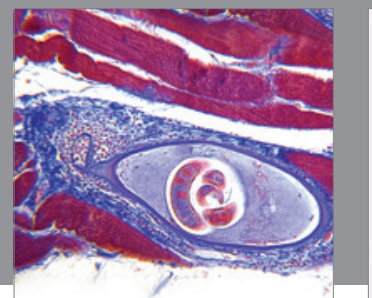

Gastroenterology

Research and Practice
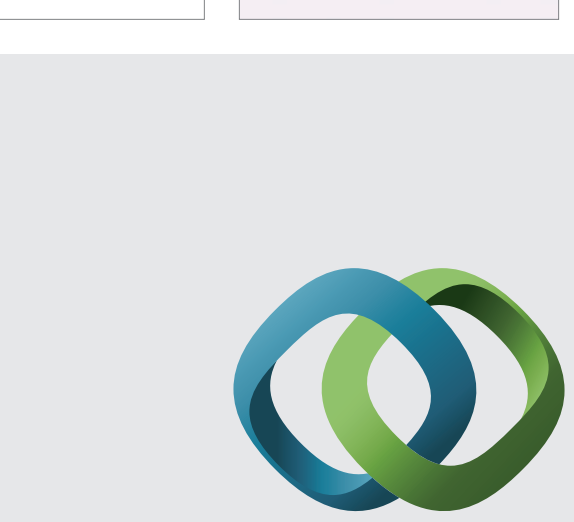

\section{Hindawi}

Submit your manuscripts at

http://www.hindawi.com
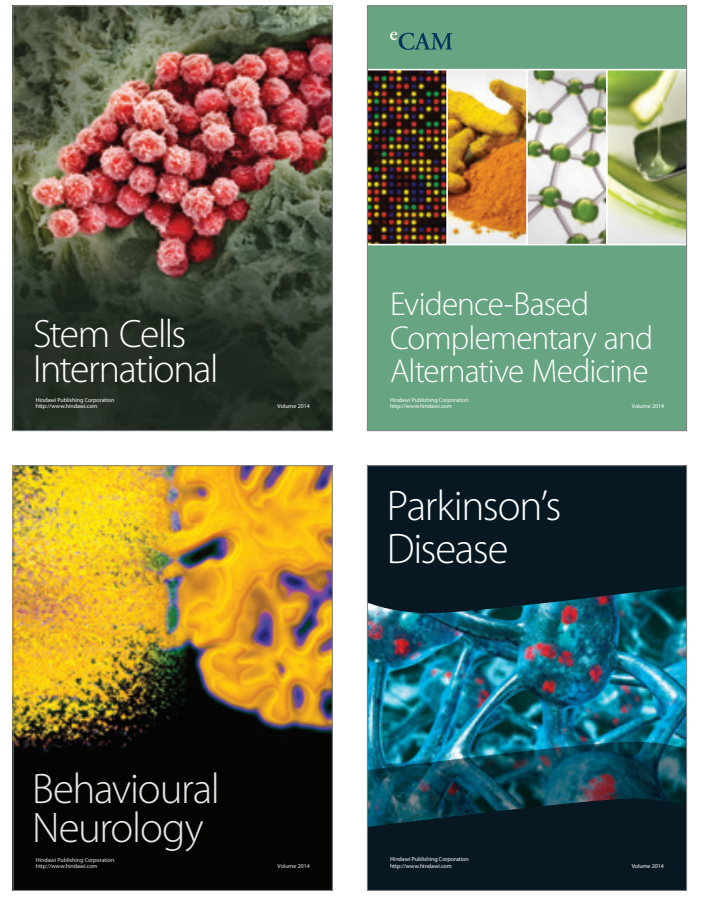
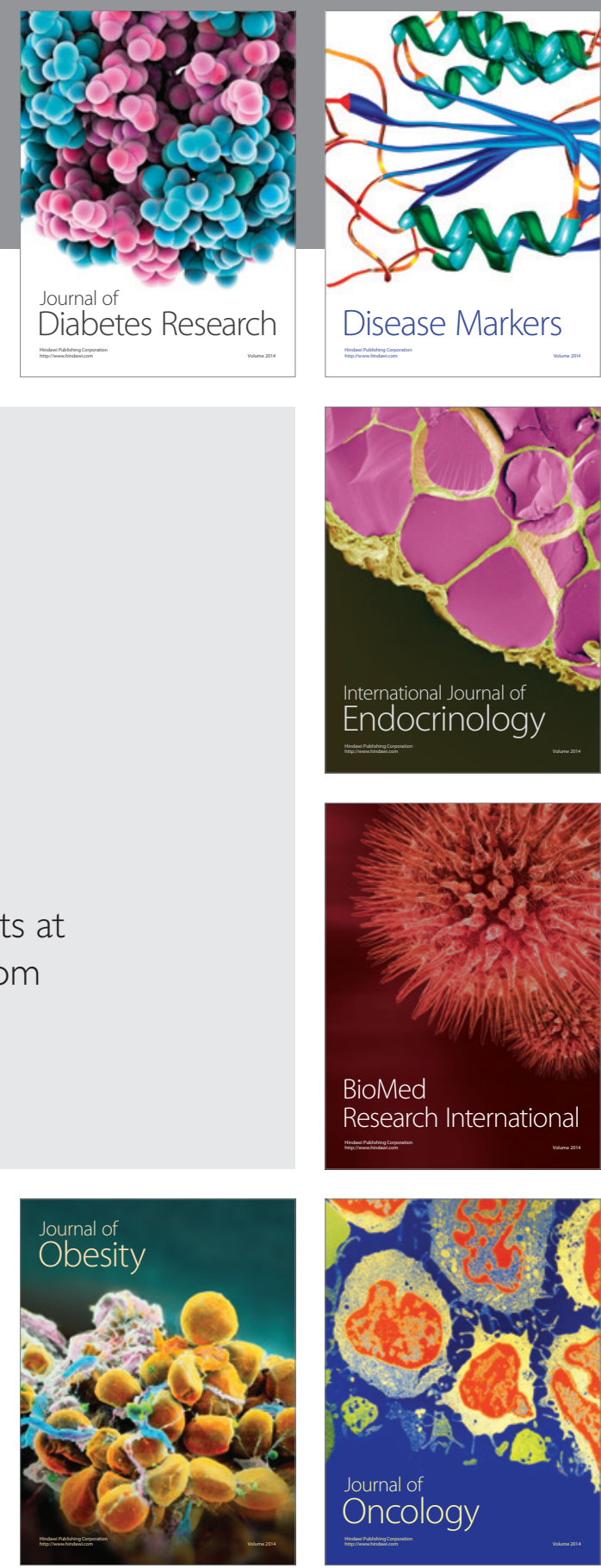

Disease Markers
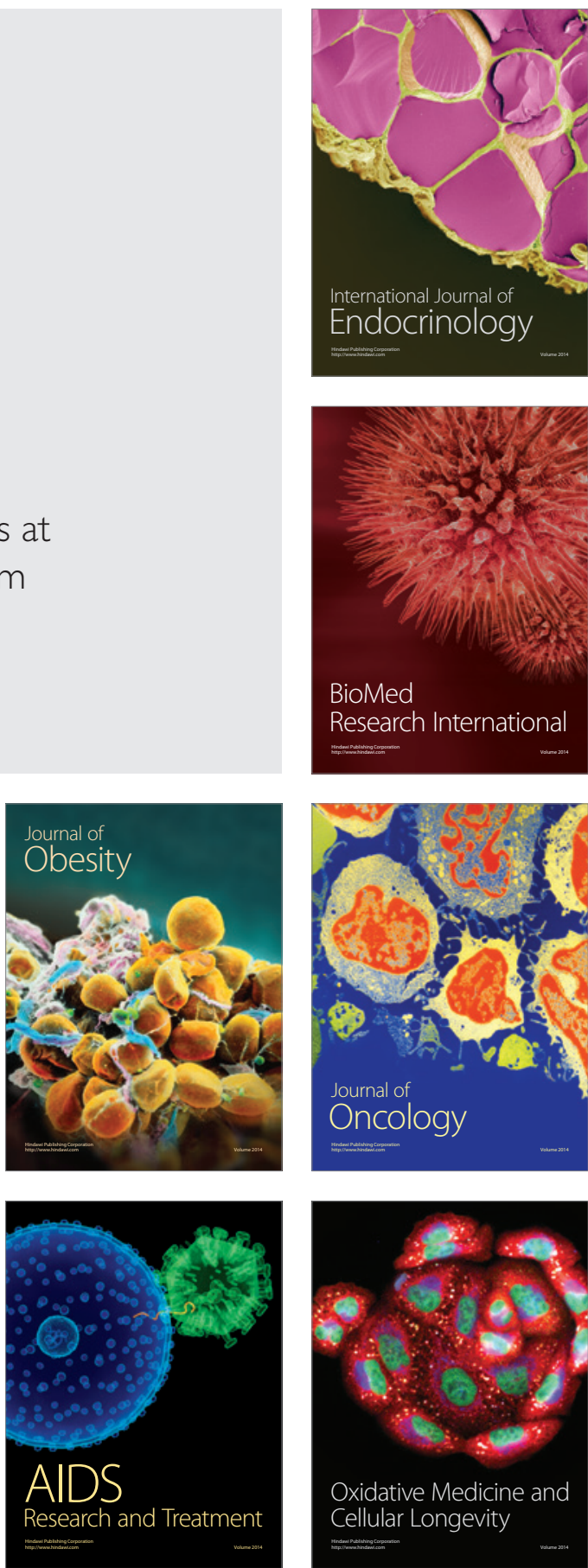